\title{
Anisotropic spectral modeling for unstably stratified homogeneous turbulence
}

\author{
Antoine Briard, ${ }^{1}$ Manasa Iyer, ${ }^{2}$ and Thomas Gomez ${ }^{2,3, *}$ \\ ${ }^{1}$ Institut Jean Le Rond d'Alembert, UMR No. 7190, UPMC, Sorbonne Universités, F-75005, Paris, France \\ ${ }^{2}$ Université Lille Nord de France, F-59000 Lille, France \\ ${ }^{3}$ LML, USTL, F-59650 Villeneuve d'Ascq, France \\ (Received 23 December 2016; published 25 April 2017)
}

\begin{abstract}
In this work, a spectral model is derived to investigate numerically unstably stratified homogeneous turbulence (USHT) at large Reynolds numbers. The modeling relies on an earlier work for passive scalar dynamics [Briard et al., J. Fluid Mech. 799, 159 (2016)] and can handle both shear and mean scalar gradients. The extension of this model to the case of active scalar dynamics is the main theoretical contribution of this paper. This spectral modeling is then applied at large Reynolds numbers to analyze the scaling of the kinetic energy, scalar variance, and scalar flux spectra and to study as well the temporal evolution of the mixing parameter, the Froude number, and some anisotropy indicators in USHT. A theoretical prediction for the exponential growth rate of the kinetic energy, associated with our model equations, is derived and assessed numerically. Throughout the validation part, results are compared with an analogous approach, restricted to axisymmetric turbulence, which is more accurate in term of anisotropy description, but also much more costly in terms of computational resources [Burlot et al., J. Fluid Mech. 765, 17 (2015)]. It is notably shown that our model can qualitatively recover all the features of the USHT dynamics, with good quantitative agreement on some specific aspects. In addition, some remarks are proposed to point out the similarities and differences between the physics of USHT, shear flows, and passive scalar dynamics with a mean gradient, the two latter configurations having been addressed previously with the same closure. Moreover, it is shown that the anisotropic part of the pressure spectrum in USHT scales in $k^{-11 / 3}$ in the inertial range, similarly to the one in shear flows. Finally, at large Schmidt numbers, a different spectral range is found for the scalar flux: It first scales in $k^{-3}$ around the Kolmogorov scale and then further in $k^{-1}$ in the viscous-convective range.
\end{abstract}

DOI: 10.1103/PhysRevFluids.2.044604

\section{INTRODUCTION}

Rayleigh-Taylor instability is a phenomenon that occurs for fluid of variable density. This instability can be found in various areas, such as geophysical, astrophysical, and confined industrial flows [1,2]. More specifically, the Rayleigh-Taylor instability can happen in natural flows such as atmospheric ones because of the mean vertical temperature gradient, when the heavy fluid, located above the lighter one, pushes it downward due to gravitational acceleration, which creates a mixing zone.

To investigate both numerically and theoretically a mechanism as complex as Rayleigh-Taylor instability, it is convenient to work in the framework of unstably stratified homogeneous turbulence (USHT), which notably discards inhomogeneity, considers the turbulent fluctuations of the velocity and buoyant fields, and uses the Boussinesq approximation to reflect the retroaction of the convected buoyant field on the velocity one [3-6]. In particular, such an idealized configuration permits one to study the time evolution of the turbulent fluctuations through second-order moments such as the kinetic energy $K=\left\langle u_{i} u_{i}\right\rangle / 2$, where $u_{i}$ is the fluctuating velocity field, and the repartition of anisotropy in the flow by the means of dedicated spectra. Furthermore, for simplicity and for

\footnotetext{
*thomas.gomez@univ-lille1.fr
} 
comparisons purposes, we consider that the stratification frequency $N$ is constant. We note that frameworks different from USHT can be considered as well, for instance, variable-density flows where the Boussinesq approximation is not used anymore $[7,8]$.

A deep understanding of unstably stratified turbulence is of great interest: from a practical point of view, for the improvement of one-point models by having an accurate knowledge of the global dynamics, and for theoretical considerations as well, when it comes to the investigation of asymptotic anisotropic states at large Reynolds numbers. These different features have been addressed recently with an axisymmetric eddy-damped quasinormal Markovian (EDQNM) model developed by Burlot and co-workers [4-6] to analyze the large Reynolds numbers dynamics of USHT. In what follows, this model will be referred to as the axisymmetric EDQNM model. As specified by its name, this spectral approach is dedicated to axisymmetric configurations and permits an accurate investigation of the scale-by-scale anisotropy distribution and of the time evolution of one-point statistics such as the Froude number Fr and the mixing intensity $\Lambda$, which will be defined later on.

However, this axisymmetric EDQNM model cannot handle, at least in the present form, shear flows, where there is no particular symmetry. For this reason, we propose an alternative method to investigate the asymptotic states at large Reynolds numbers of USHT. Our method is also based on the EDQNM procedure classically used in homogeneous and isotropic turbulence (HIT) [9,10], but in addition a departure from isotropy is modeled through spherically averaged spectra that measure both directional and polarization anisotropies [11]. Basically, spectral second-order correlations of interest, namely, the velocity-velocity, scalar-scalar, and mixed velocity-scalar two-point correlations, are expanded into spherical harmonics and this expansion is further truncated at the second order. Angular information about anisotropy is then partially recovered due to spectral anisotropy indicators. This two-step method (the classical EDQNM model combined with a model for anisotropy), referred to as anisotropic EDQNM modeling, developed by Mons et al. [12] and further applied by Briard et al. [13], was first used to study shear-driven turbulence, such as sustained shear flows, axisymmetric contractions and expansions, shear-released turbulence, and plane distortion. Afterward, the anisotropic EDQNM modeling was consistently extended to handle the transport of a passive scalar field by Briard et al. [14], to deal in particular with turbulence submitted to both shear and mean scalar gradients. In the latter reference, a configuration of interest is tackled: homogeneous isotropic turbulence with a mean scalar gradient (HITSG), which is the counterpart of USHT for a passive scalar field instead of an active one. The anisotropic EDQNM modeling was successfully assessed by comparisons with several direct numerical simulations (DNSs) and experiments in Refs. [12-14]. Thus, it can be used in multiple configurations, but only axisymmetry will be considered here.

Consequently, it is proposed in the present work to extend theoretically this spectral model to the case of active scalar dynamics with USHT and to compare it to the results obtained with the axisymmetric EDQNM model of Burlot and co-workers. Throughout this paper, USHT will be qualitatively compared to results obtained with the same anisotropic EDQNM closure in the frameworks of pure shear flows and HITSG: Similarities and differences will be pointed out and the physics behind it will be discussed as well. Pressure spectra, with an anisotropic part resulting from stratification, are also addressed.

The extension of the anisotropic EDQNM modeling to unstable stratification is a step further towards the modeling and understanding of high-Reynolds-number geophysical flows, such as atmospheric and oceanic ones. Indeed, under the assumption of homogeneity, such flows contain the effects of shear, temperature, and concentration gradients, stratification, rotation, and helicity. Shear and temperature gradient mechanisms have already been addressed with our model [14] and helicity is currently the topic of another work [15], so stratification appears to be an natural extension, whereas the effects of rotation were addressed independently with another approach [16-18]. Indeed, rotating turbulence, as well as stably stratified turbulence, requires a more sophisticated EDQNM model that can take into account the effects of dispersive waves on the dynamics of the three-point third-order correlations.

Finally, the present model is used to address, from a fundamental point of view, the effects of large Schmidt numbers Sc on the inertial scaling of the scalar flux spectrum, which differs from passive 
scalar dynamics [19], where Sc is the ratio of kinematic viscosity $v$ to the molecular diffusivity $a$. Despite the theoretical interest in determining the asymptotic regimes of USHT at large Reynolds and Schmidt numbers, this framework represents some practical interests when it comes to the modeling of oceanic flows, where unstable stratification can results from double diffusion processes [20] with saltwater $\left(10^{2} \leqslant \mathrm{Sc} \leqslant 10^{3}\right)$. Indeed, at the ocean surface, hot salty water is on top of cooler and saltier water, so the stratification is stable; however, when the temperature drops off in the air layer above the ocean, the upper salty water cools down very rapidly, because heat transfers are much more efficient than mass transfers. In the end, one has a heavier fluid on top, causing the appearance of unstably stratified water columns [21].

The paper is structured as follows. The main features of the original anisotropic EDQNM modeling and the extension to active scalar dynamics are presented in Sec. II. Some additional theoretical considerations about the modeling are proposed in the Appendix. The model is applied to investigate the large-Reynolds-number asymptotic anisotropic states of USHT in Sec. III. More precisely, scalings of spectra are addressed in the infrared and inertial ranges, along with the scale-by-scale distribution of anisotropy and the time evolution of one-point statistics. This section should be seen as an advanced validation of our approach since multiple qualitative and quantitative comparisons with the axisymmetric EDQNM model are made. In addition, further insights are proposed while comparing the dynamics of USHT with the one of HITSG and shear flows, configurations that have been consistently investigated with the same closure. Applications of our modeling are finally exposed in Sec. IV. The emphasis is put on pressure spectra in Sec. IV A to underline the strong analogy with shear flows. The effects of large Schmidt numbers Sc on spectral scalings and on the dynamics of USHT are revealed in Sec. IV B. A summary is given and results are discussed in Sec. V.

\section{SPECTRAL MODELING FOR USHT}

In this section the spectral model for passive scalar dynamics developed in Ref. [14] is consistently extended to the case of an active scalar for unstably stratified turbulence. The extension is rather simple, because stratification amounts to two additional production terms with respect to the case of passive scalar fluctuations in HITSG: a retroaction of the spectral scalar-scalar correlation on the scalar flux equation and similarly a retroaction of the scalar flux on the spectral velocity-velocity correlation equation. This strong coupling notably causes the kinetic energy, scalar variance, and mixed correlation to grow exponentially at the same rate, which is fundamentally different from passive scalar dynamics in HITSG, where the scalar variance increases algebraically whereas the mixed correlation and kinetic energy both decay algebraically.

\section{A. Main equations of USHT}

In this section the exact evolution equations for the second-order spectral correlations are derived. First, as commonly done for USHT, one can scale the scalar fluctuations $\theta$, which can be, for instance, a concentration, as a buoyant velocity $c[3]$ according to

$$
c=\frac{2 \mathcal{A} g \theta}{N}
$$

where $N$ is the stratification characteristic time, or buoyancy frequency

$$
N=\sqrt{2 \mathcal{A g} \frac{d \Theta}{d x_{3}}},
$$

with $g$ the gravitational acceleration, $\Theta$ the mean scalar field, and $\mathcal{A}$ the Atwood number $\mathcal{A}=$ $\left(\rho_{1}-\rho_{2}\right) /\left(\rho_{1}+\rho_{2}\right)$, where $\rho_{1}$ and $\rho_{2}$ are the densities of the heavy and light fluids, respectively. In addition, $N$ is expressed in units of $\tau_{0}^{-1}$, where $\tau_{0}$ is the characteristic eddy turnover time. Further, $N$ should be compared qualitatively to the shear intensity $S$ in shear flows, so that here for USHT, 
$N t$ represents the relevant dimensionless time. The mean gradient is chosen to be vertical (along $x_{3}$ ) so that the evolution equations of the fluctuating velocity and scalar fields are

$$
\begin{gathered}
\frac{\partial c}{\partial t}+u_{j} \frac{\partial c}{\partial x_{j}}=a \frac{\partial^{2} c}{\partial x_{j} \partial x_{j}}+N u_{3}, \\
\frac{\partial u_{i}}{\partial t}+u_{j} \frac{\partial u_{i}}{\partial x_{j}}=-\frac{\partial p}{\partial x_{i}}+v \frac{\partial^{2} u_{i}}{\partial x_{j} \partial x_{j}}+N c \delta_{i 3},
\end{gathered}
$$

where $v$ is the kinematic viscosity and $a$ the scalar diffusivity. The spectral counterpart of these equations are straightforward and can be found, for instance, in Ref. [4].

The three two-point second-order spectral correlations of interest are as follows: the spectral Reynolds tensor $\hat{R}_{i j}$, or velocity-velocity correlation; the scalar-scalar correlation $\mathcal{E}^{T}$; and the scalar flux $F_{i}$, or mixed velocity-scalar correlation. Their respective definitions are

$$
\begin{gathered}
\hat{R}_{i j}(\boldsymbol{k}, t) \delta(\boldsymbol{k}-\boldsymbol{p})=\left\langle\hat{u}_{i}^{*}(\boldsymbol{p}, t) \hat{u}_{j}(\boldsymbol{k}, t)\right\rangle, \\
\mathcal{E}^{T}(\boldsymbol{k}, t) \delta(\boldsymbol{k}-\boldsymbol{p})=\left\langle\hat{c}^{*}(\boldsymbol{p}, t) \hat{c}(\boldsymbol{k}, t)\right\rangle, \\
F_{i}(\boldsymbol{k}, t) \delta(\boldsymbol{k}-\boldsymbol{p})=\left\langle\hat{u}_{i}^{*}(\boldsymbol{p}, t) \hat{c}(\boldsymbol{k}, t)\right\rangle,
\end{gathered}
$$

where a caret denotes the Fourier transform, an asterisk denotes the complex conjugate, $\hat{u}_{i}$ and $\hat{c}$ are the spectral fluctuating velocity and scalar fields, respectively, and $\boldsymbol{k}$ and $\boldsymbol{p}$ are wave vectors. These definitions are similar to the ones given in Ref. [14], except that here the scalar $\hat{\theta}(\boldsymbol{k})$ has been replaced by the spectral buoyant velocity $\hat{c}(\boldsymbol{k})$. The exact evolution equations of these correlations $\hat{R}_{i j}, F_{i}$, and $\mathcal{E}^{T}$ read

$$
\begin{gathered}
\left(\frac{\partial}{\partial t}+2 \nu k^{2}\right) \hat{R}_{i j}(\boldsymbol{k}, t)=T_{i j}^{\mathrm{NL}}(\boldsymbol{k})+\underbrace{N\left[P_{j 3} F_{i}(\boldsymbol{k}, t)+P_{i 3} F_{j}(\boldsymbol{k}, t)\right]}_{\text {additional coupling }}, \\
\left(\frac{\partial}{\partial t}+(v+a) k^{2}\right) F_{i}(\boldsymbol{k}, t)=T_{i}^{\mathrm{F}, \mathrm{NL}}(\boldsymbol{k}, t)+N \hat{R}_{i 3}(\boldsymbol{k}, t)+\underbrace{N P_{i 3} \mathcal{E}^{T}(\boldsymbol{k}, t)}_{\text {additional coupling }}, \\
\left(\frac{\partial}{\partial t}+2 a k^{2}\right) \mathcal{E}^{T}(\boldsymbol{k}, t)=T^{\mathrm{T}, \mathrm{NL}}(\boldsymbol{k}, t)+2 N F_{3}(\boldsymbol{k}, t),
\end{gathered}
$$

where $T_{i j}^{\mathrm{NL}}, T_{i}^{\mathrm{F}, \mathrm{NL}}$ and $T^{\mathrm{T}, \mathrm{NL}}$ are the exact spectral nonlinear transfers, which can be expressed as functions of three-point triple correlations, and $P_{i j}=\delta_{i j}-\alpha_{i} \alpha_{j}$ is a projector, with $\alpha_{i}=k_{i} / k$. These evolution equations are exact, and the terms labeled "additional coupling" refer to the supplementary production terms arising from stratification, compared to HITSG, which were mentioned previously. In the next section the main features of the modeling developed in Ref. [14] for passive scalar dynamics are recalled and the final spherically averaged evolution equations of USHT are consistently derived.

\section{B. Modeling of anisotropy: Link with the axisymmetric EDQNM model}

The previous spectral evolution equations (8)-(10) are exact and the two-step anisotropic EDQNM modeling method of $[12,14]$ is now applied. The first step consists into closing the nonlinear terms and the second one into modeling anisotropy through spherically averaged spectra. All the details are gathered in Refs. [12,14] and the main elements are given here for the sake of clarity. We first recall the principal features of the anisotropy modeling and the decomposition of the spectral second-order correlations. Considerations about the classical EDQNM procedure, i.e., the closure of nonlinear terms, are discussed after the final spherically averaged evolution equations. 
The starting point is the optimal trace-deviator intrinsic decomposition of $\hat{R}_{i j}$ in terms of isotropic, directional, and polarization parts [11,12]

$$
\hat{R}_{i j}(\boldsymbol{k}, t)=\underbrace{\frac{E(k, t)}{4 \pi k^{2}} P_{i j}(\boldsymbol{k})}_{\hat{R}_{i j}^{\text {(iso) }}(\boldsymbol{k}, t)}+\underbrace{\left(\mathcal{E}(\boldsymbol{k}, t)-\frac{E(k, t)}{4 \pi k^{2}}\right) P_{i j}(\boldsymbol{k})}_{\hat{R}_{i j}^{\text {(dir) }}(\boldsymbol{k}, t)}+\underbrace{\operatorname{Re}\left[Z(\boldsymbol{k}, t) N_{i}(\boldsymbol{k}) N_{j}(\boldsymbol{k})\right]}_{\hat{R}_{i j}^{(\text {pol }}(\boldsymbol{k}, t)},
$$

with $E(k, t)$ the kinetic energy spectrum

$$
E(k, t)=\int_{S_{k}} \frac{1}{2} \hat{R}_{i i}(\boldsymbol{k}, t) d^{2} \boldsymbol{k}=\int_{S_{k}} \mathcal{E}(\boldsymbol{k}, t) d^{2} \boldsymbol{k},
$$

where $\mathcal{E}(\boldsymbol{k}, t)$ refers to the directional anisotropy and measures the departure from isotropy along the wave vector $\boldsymbol{k} ; Z(\boldsymbol{k}, t)$ reflects the polarization anisotropy, i.e., the difference of energy between the components of the spectral Reynolds tensor, and $N_{i}$ are helical modes [16,22].

Then $\hat{R}_{i j}$, and more precisely $\mathcal{E}$ and $Z$, are expanded into spherical harmonics in order to compute anisotropic descriptors that depend only on the wave-number modulus $k$ and no longer on the orientation of the vector $\boldsymbol{k}$, which dramatically reduces the computational time. The expansion in spherical harmonics is further truncated at the second order: Consequences of such a truncation and validations of this approximation are thoroughly presented and discussed in Refs. [12-14]. The spectral anisotropy descriptors $H_{i j}^{(\mathrm{dir})}$ and $H_{i j}^{(\mathrm{pol})}$ resulting from this modeling are symmetric deviatoric tensors, linked to directional and polarization anisotropies, respectively, obtained by integrating the second-order expansion of $\hat{R}_{i j}$ on the sphere $S_{k}$ of radius $k$,

$$
\begin{aligned}
& 2 E(k, t) H_{i j}^{(\mathrm{dir})}(k, t)=\int_{S_{k}} \hat{R}_{i j}^{(\mathrm{dir})}(\boldsymbol{k}, t) d^{2} \boldsymbol{k}, \\
& 2 E(k, t) H_{i j}^{(\mathrm{pol})}(k, t)=\int_{S_{k}} \hat{R}_{i j}^{(\mathrm{pol})}(\boldsymbol{k}, t) d^{2} \boldsymbol{k} .
\end{aligned}
$$

From the previous equations, one can determine the spectral tensor

$$
\phi_{i j}(k, t)=\int_{S_{k}} \hat{R}_{i j}(\boldsymbol{k}, t) d^{2} \boldsymbol{k}=2 E(k, t)\left(\frac{\delta_{i j}}{3}+H_{i j}^{(\mathrm{dir})}(k, t)+H_{i j}^{(\mathrm{pol})}(k, t)\right) .
$$

A similar procedure was applied in Ref. [14] to extract from the spectral scalar-scalar correlation its directional anisotropy part, which yielded the definition of $H_{i j}^{(T)}$, equivalent to $H_{i j}^{(\mathrm{dir})}$ for the velocity field

$$
2 E_{T}(k, t) H_{i j}^{(T)}(k, t)=\int_{S_{k}}\left(\mathcal{E}^{T}(\boldsymbol{k}, t)-\frac{E_{T}(k, t)}{4 \pi k^{2}}\right) P_{i j}(\boldsymbol{k}) d^{2} \boldsymbol{k},
$$

where $E_{T}(k, t)$ is the scalar variance spectrum, which can also be called the buoyancy spectrum in USHT, given by

$$
E_{T}(k, t)=\int_{S_{k}} \mathcal{E}^{T}(\boldsymbol{k}, t) d^{2} \boldsymbol{k}
$$

Obviously, there is no polarization anisotropy for the buoyant field since it is a scalar quantity. Interestingly, $H_{i j}^{(T)}$, which is specific to the anisotropic EDQNM modeling developed in Ref. [14], can be linked to another indicator of anisotropy often used [4-6], which could be called a scale-by-scale dimensionality parameter

$$
\sin ^{2} \gamma_{T}(k, t)=\frac{1}{E_{T}(k, t)} \int_{S_{k}} \sin ^{2} \theta_{k}(\boldsymbol{k}) \mathcal{E}^{T}(\boldsymbol{k}, t) d^{2} \boldsymbol{k}=2 H_{33}^{(T)}(k, t)+\frac{2}{3},
$$

where $\theta_{k}(\boldsymbol{k})$ is the angle between the vertical axis (here $x_{3}$ ) and the wave vector $\boldsymbol{k}$ : $\theta_{k}$ is sketched in Fig. 1. When the considered scales are isotropic, one has $\sin ^{2} \gamma_{T}=2 / 3$ and $H_{i j}^{(T)}=0$. Then the 


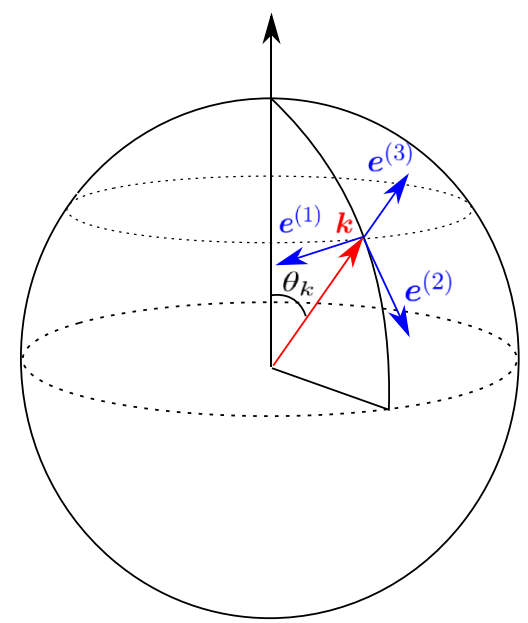

FIG. 1. Craya-Herring frame $\left(\boldsymbol{e}^{(\mathbf{1})}, \boldsymbol{e}^{(\mathbf{2})}, \boldsymbol{e}^{(3)}\right)$ in blue and the wave vector $\boldsymbol{k}$ in red. The fluctuating spectral velocity $\hat{\boldsymbol{u}}$ is contained in the plane $\left(\boldsymbol{e}^{(\mathbf{1})}, \boldsymbol{e}^{(\mathbf{2})}\right)$.

scalar flux is decomposed as

$$
F_{i}(\boldsymbol{k}, t)=\frac{3}{2} \frac{E_{j}^{F}(k, t)}{4 \pi k^{2}} P_{i j}(\boldsymbol{k}),
$$

as defined in Refs. $[14,23,24]$. The spectrum $E_{j}^{F}$ is real and is the spherical average of the scalar flux according to

$$
E_{i}^{F}(k, t)=\int_{S_{k}} F_{i}(\boldsymbol{k}, t) d^{2} \boldsymbol{k} .
$$

Since the only nonzero component of the scalar flux is the component along the mean gradient, we further define the cospectrum as $\mathcal{F}=E_{3}^{F}$, as in Ref. [14] for HITSG.

Before presenting the final spherically averaged evolution equations of the present anisotropic EDQNM modeling, we expose the equations solved numerically in Ref. [4]. From the exact evolution equation of $\hat{R}_{i j}$ and the decomposition (11), one obtains exact equations for $\mathcal{E}$ and $Z$. For axisymmetric turbulence without helicity, these two quantities can be related to the toroidal and poloidal potentials $\mathcal{E}^{\text {(toro) }}$ and $\mathcal{E}^{\text {(polo) }}$ through

$$
\mathcal{E}(\boldsymbol{k})=\mathcal{E}^{(\text {polo })}(\boldsymbol{k})+\mathcal{E}^{\text {(toro) }}(\boldsymbol{k}), \quad Z(\boldsymbol{k})=\mathcal{E}^{(\text {polo) }}(\boldsymbol{k})-\mathcal{E}^{(\text {toro })}(\boldsymbol{k}) .
$$

These potentials $\mathcal{E}^{\text {(toro) }}$ and $\mathcal{E}^{\text {(polo) }}$ are defined as

$$
\begin{aligned}
\mathcal{E}^{(\text {toro })}(\boldsymbol{k}) \delta(\boldsymbol{k}-\boldsymbol{p}) & =\left\langle\hat{u}^{\text {(toro }) *}(\boldsymbol{p}) \hat{u}^{(\text {toro })}(\boldsymbol{k})\right\rangle, \\
\mathcal{E}^{(\text {polo })}(\boldsymbol{k}) \delta(\boldsymbol{k}-\boldsymbol{p}) & =\left\langle\hat{u}^{(\text {polo }) *}(\boldsymbol{p}) \hat{u}^{(\text {polo })}(\boldsymbol{k})\right\rangle,
\end{aligned}
$$

where the spectral fluctuating velocity is

$$
\hat{u}_{i}(\boldsymbol{k})=\hat{u}^{(\text {toro })}(\boldsymbol{k}) e_{i}^{(1)}(\boldsymbol{k})+\hat{u}^{(\text {polo })}(\boldsymbol{k}) e_{i}^{(2)}(\boldsymbol{k}),
$$

in the Craya-Herring frame $\left(\boldsymbol{e}^{(1)}, \boldsymbol{e}^{(2)}, \boldsymbol{k}\right)[22,25]$, illustrated in Fig. 1. All the details for the linkage of the Craya-Herring decomposition to its toroidal-poloidal counterpart are given in Ref. [22], as well as its connection to various wave-vortex decompositions, which are of physical interest for rotating stably stratified turbulence. Indeed, it separates the nonpropagating and propagating parts of the flow, the latter being linked to inertial-gravity waves. 
In the axisymmetric EDQNM model of Burlot and co-workers, the evolution equations of $\mathcal{E}^{\text {(toro) }}$, $\mathcal{E}^{\text {(polo) }}, \mathcal{E}^{T}$, and $F_{3}$ are solved. Their production terms, which depend linearly on the stratification frequency $N$, are exact and a classical EDQNM procedure is used to close the triple correlations. The toroidal and poloidal potentials can be related to the present deviatoric tensors $H_{i j}^{(\mathrm{dir})}$ and $H_{i j}^{(\mathrm{pol})}$ appearing through the modeling of anisotropy $[12,14]$. After some algebra and the truncation at the second order, one gets

$$
\mathcal{E}^{(\mathrm{dir})}\left(\theta_{k}, k\right)=-\frac{15}{2} \mathcal{E}_{0}(k) H_{33}^{(\mathrm{dir})}(k)\left(3 \cos ^{2} \theta_{k}-1\right), \quad Z\left(\theta_{k}, k\right)=\frac{15}{2} \mathcal{E}_{0}(k) H_{33}^{(\mathrm{pol})}(k) \sin ^{2} \theta_{k},
$$

where $\mathcal{E}_{0}=E / 4 \pi k^{2}$ and $\mathcal{E}^{(\mathrm{dir})}=\mathcal{E}-\mathcal{E}_{0}$, so that

$$
\begin{aligned}
& \mathcal{E}^{\text {(toro) }}\left(\theta_{k}, k\right)=\frac{\mathcal{E}_{0}(k)}{2}\left[1-\frac{15}{2}\left[H_{33}^{(\mathrm{pol})}(k) \sin ^{2} \theta_{k}+H_{33}^{(\mathrm{dir})}(k)\left(3 \cos ^{2} \theta_{k}-1\right)\right]\right] \\
& \mathcal{E}^{(\text {polo })}\left(\theta_{k}, k\right)=\frac{\mathcal{E}_{0}(k)}{2}\left[1+\frac{15}{2}\left[H_{33}^{(\mathrm{pol})}(k) \sin ^{2} \theta_{k}-H_{33}^{(\mathrm{dir})}(k)\left(3 \cos ^{2} \theta_{k}-1\right)\right]\right] .
\end{aligned}
$$

So far in this section, the relations between the present approach using a model for anisotropy and the axisymmetric EDQNM model were given in a manner similar to [26]. In the next section the final spherically averaged equations of the present anisotropic EDQNM modeling are presented, along with the additional production terms mentioned earlier. As a conclusion to this section, we remark that injecting (26) and (27), resulting from the present modeling, in the equations of $\mathcal{E}^{\text {(toro) }}$ and $\mathcal{E}^{\text {(polo) }}$ solved in the axisymmetric EDQNM model would yield the final equations presented hereafter.

\section{Final anisotropic EDQNM modeling for USHT}

In the previous exact evolution equations (8)-(10), the nonlinear transfer terms are closed by a classical EDQNM procedure: Third-order correlations are expressed as functions of the spectral second-order moments $\hat{R}_{i j}, \mathcal{E}^{T}$, and $F_{i}$. Finally, using the definitions of $H_{i j}^{(\mathrm{dir})}, H_{i j}^{(\mathrm{pol})}, H_{i j}^{(T)}$, and $\mathcal{F}$, which result from the modeling of anisotropy, one obtains the final spherically averaged Lin equations for USHT

$$
\begin{gathered}
\left(\frac{\partial}{\partial t}+2 v k^{2}\right) E(k, t)=S^{\mathrm{NL}(\mathrm{iso})}(k, t)+S^{\mathrm{L}, \mathrm{USHT}(\mathrm{iso})}(k, t), \\
\left(\frac{\partial}{\partial t}+2 v k^{2}\right) E(k, t) H_{33}^{\text {(dir) }}(k, t)=S_{33}^{\mathrm{NL}(\mathrm{dir})}(k, t)+S_{33}^{\mathrm{L}, \mathrm{USHT}(\mathrm{dir})}(k, t), \\
\left(\frac{\partial}{\partial t}+2 v k^{2}\right) E(k, t) H_{33}^{(\mathrm{pol})}(k, t)=S_{33}^{\mathrm{NL}(\mathrm{pol})}(k, t)+S_{33}^{\mathrm{L}, \mathrm{USHT}(\mathrm{pol})}(k, t), \\
\left(\frac{\partial}{\partial t}+2 a k^{2}\right) E_{T}(k, t)=S^{\mathrm{T}, \mathrm{NL}(\mathrm{iso})}(k, t)+2 N \mathcal{F}(k, t), \\
\left(\frac{\partial}{\partial t}+2 a k^{2}\right) E_{T}(k, t) H_{33}^{(T)}(k, t)=S_{33}^{\mathrm{T}, \mathrm{NL}(\mathrm{dir})}(k, t)+\frac{2}{15} N \mathcal{F}(k, t), \\
\left(\frac{\partial}{\partial t}+(v+a) k^{2}\right) \mathcal{F}(k, t)=S_{3}^{\mathrm{F}, \mathrm{NL}}(k, t)+N \phi_{33}(k, t)+S_{3}^{\mathrm{F}, \mathrm{L}, \mathrm{USHT}}(k, t) .
\end{gathered}
$$

Some remarks need to be said about these equations. First, only the $H_{33}^{()}$component along the mean gradient is involved, because USHT is an axisymmetric configuration, so there are no extra-diagonal components. In addition, because of incompressibility and symmetry, the $H_{i j}^{()}$tensors are trace-free, so $H_{11}^{()}=H_{22}^{()}=-H_{33}^{()} / 2$. Hence, in terms of equations to solve simultaneously, USHT is simpler that a passive scalar advected by a turbulent shear flow, where sixteen independent equations need to be solved, instead of six here. 
On the right-hand sides of Eqs. (28)-(33), the first terms with the upper index ( $)^{\mathrm{NL}}$ refer to the spherically averaged nonlinear transfers, which are exactly the same as in passive scalar dynamics, whose explicit formulas are gathered in Ref. [14]. The fact that the nonlinear transfers are identical for both passive and active scalar dynamics is because the linear operators, of the equations for the three-point third-order correlations, are not taken into account, consistently with $[12,14]$. The other right-hand-side terms of Eqs. (28)-(33) are the linear spherically averaged transfers, or production terms linked to the mean scalar gradient, which can be divided into two parts: the new ones written with the upper index ()$^{\mathrm{L} \text {,USHT }}$ and the ones already present in $\operatorname{HITSG}\left(2 N \mathcal{F}, 2 N \mathcal{F} / 15\right.$, and $\left.N \phi_{33}\right)$. The former terms come from the extension of the anisotropic EDQNM modeling for passive scalar dynamics to the case of unstable stratification and as such represent the main theoretical contribution of this work. Here are their definitions and expressions:

$$
\begin{gathered}
S^{\mathrm{L}, \mathrm{USHT}(\mathrm{iso})}(k, t)=N \int_{S_{k}} P_{i 3}(\boldsymbol{k}) F_{i}(\boldsymbol{k}, t) d^{2} \boldsymbol{k}=N \mathcal{F}(k, t), \\
S_{i j}^{\mathrm{L}, \mathrm{USHT}(\mathrm{dir})}(k, t)=\frac{N}{2} \int_{S_{k}} P_{l 3}(\boldsymbol{k}) F_{l}(\boldsymbol{k}, t) P_{i j}(\boldsymbol{k}) d^{2} \boldsymbol{k}-\frac{\delta_{i j}}{3} N \mathcal{F}(k, t) \\
=\frac{N}{20}\left[E_{i}^{F}(k, t) \delta_{j 3}+E_{j}^{F}(k, t) \delta_{i 3}-\frac{2}{3} \mathcal{F}(k, t) \delta_{i j}\right], \\
S_{i j}^{\mathrm{L}, \mathrm{USHT}(\mathrm{pol})}(k, t)=\frac{N}{4} \int_{S_{k}}\left[P_{l 3}(\boldsymbol{k}) F_{n}(\boldsymbol{k}, t)+P_{n 3}(\boldsymbol{k}) F_{l}(\boldsymbol{k}, t)\right] N_{l}^{*} N_{n}^{*} N_{i} N_{j} d^{2} \boldsymbol{k} \\
=\frac{3 N}{10}\left[E_{i}^{F}(k, t) \delta_{j 3}+E_{j}^{F}(k, t) \delta_{i 3}-\frac{2}{3} \mathcal{F}(k, t) \delta_{i j}\right], \\
S_{i}^{\mathrm{F}, \mathrm{L}, \mathrm{USHT}}(k, t)=N \int_{S_{k}} \mathcal{E}^{T}(\boldsymbol{k}, t) P_{i 3}(\boldsymbol{k}) d^{2} \boldsymbol{k}=2 N E_{T}(k, t)\left[\frac{1}{3} \delta_{i 3}+H_{i 3}^{(T)}(k, t)\right] .
\end{gathered}
$$

More specifically, one has here

$$
S_{33}^{\mathrm{L}, \mathrm{USHT}(\mathrm{dir})}(k, t)=\frac{N}{15} \mathcal{F}(k, t), \quad S_{33}^{\mathrm{L}, \mathrm{USHT}(\mathrm{pol})}(k, t)=\frac{2 N}{5} \mathcal{F}(k, t) .
$$

Consequently, because of our anisotropic modeling, the set of equations (28)-(33) is different from the one of Burlot and co-workers and this will have strong theoretical impact, notably in Sec. III B 3 for the prediction of the exponential growth rate of one-point statistics. Here are the definitions of the important one-point statistics, computed from spherically averaged spectra, such as kinetic energy, scalar variance, mixed correlation, and their respective dissipation rates

$$
\begin{gathered}
K(t)=\frac{1}{2}\left\langle u_{i} u_{i}\right\rangle=\int_{0}^{\infty} E(k, t) d k, \quad \epsilon(t)=2 v \int_{0}^{\infty} k^{2} E(k, t) d k, \\
K_{T}(t)=\left\langle\theta^{2}\right\rangle=\int_{0}^{\infty} E_{T}(k, t) d k, \quad \epsilon_{T}(t)=2 a \int_{0}^{\infty} k^{2} E_{T}(k, t) d k, \\
K_{\mathcal{F}}(t)=\left\langle u_{3} \theta\right\rangle=\int_{0}^{\infty} \mathcal{F}(k, t) d k, \quad \epsilon_{\mathcal{F}}(t)=(v+a) \int_{0}^{\infty} k^{2} \mathcal{F}(k, t) d k .
\end{gathered}
$$

In the next section basic elements of our numerical simulations are exposed.

\section{Numerical setup}

Some numerical aspects of our simulations are briefly presented here. The six previous spherically averaged Lin equations of USHT are solved using a third-order Runge-Kutta scheme with implicit treatment of diffusion terms. The wave-number space is discretized using a logarithmic mesh $k_{i+1}=10^{1 / f} k_{i}$, where $f=15$ is the number of points per decade. This mesh spans from $k_{\min }$ to $k_{\max }$, where $k_{\max }=10 k_{\eta}$ for $\mathrm{Sc}=1$ and $k_{\max }=10 k_{B}$ for $\mathrm{Sc} \gg 1$, where $k_{\eta}=\left(\epsilon / \nu^{3}\right)^{1 / 4}$ is the Kolmogorov 
wave number and $k_{B}=\sqrt{\mathrm{Sc}} k_{\eta}$ is the Batchelor wave number. The time step is monitored by defining a constant Courant-Friedrichs-Lewy number and is obtained by considering the characteristic time scales of scalar and kinetic dynamics.

Two types of large-scale initial conditions are mainly addressed: Saffman and Batchelor turbulence, which correspond, respectively, to infrared slopes $\sigma=2$ and $\sigma=4$, where $E(k<$ $\left.k_{L}\right) \sim k^{\sigma}$, with $k_{L}$ the integral wave number. We define as well in a similar manner the scalar infrared slope $\sigma_{T}: E_{T}\left(k<k_{T}\right) \sim k^{\sigma_{T}}$, with $k_{T}$ the peak of the scalar variance spectrum. The initial conditions are isotropic, meaning that $\mathcal{F}(k, t=0)=0$, and are borrowed from [27]

$$
E(k, t=0)=K_{0} k^{-5 / 3} \epsilon^{2 / 3} f_{L}(k L) f_{\eta}(k \eta)
$$

where $f_{L}$ and $f_{\eta}$ are shape functions for large and small scales, respectively,

$$
\begin{aligned}
& f_{L}(x)=\left(\frac{x}{\left(x^{1.5}+1.5-\sigma / 4\right)^{2 / 3}}\right)^{5 / 3+\sigma}, \\
& f_{\eta}(x)=\exp \left\{-5.3\left[\left(x^{4}+0.4^{4}\right)^{1 / 4}-0.4\right]\right\}
\end{aligned}
$$

For simplicity, we choose $E_{T}(k, t=0)=E(k, t=0)$, and some considerations on what values should be chosen for $\sigma$ and $\sigma_{T}$ are proposed hereafter in Sec. III A. The initial Reynolds number based on the Taylor microscale is $\operatorname{Re}_{\lambda}(0) \sim 5$, and in all the present simulations, the realizability conditions derived in Refs. $[12,14]$ are always verified.

Moreover, let us specify here that in the nonlinear transfers of Eqs. (28)-(33), the eddy-damping terms are kept isotropic and the constants involved are identical, for consistency purposes, to the ones chosen in Ref. [14], meaning that $A_{1}=0.355$ for the velocity field and $A_{2}=0$ and $A_{3}=1.3$ for the scalar and scalar flux fields. This is a classical choice of constants for isotropic turbulence $[10,28]$; moreover, such a setting proved to be efficient for passive scalar dynamics in complex flows, such as turbulent shear flows with a mean scalar gradient. This further justifies the choice of the authors to keep the constants as in Ref. [14]. Interestingly, in Refs. [4,5], the choice of eddy-damping constants is $A_{1}=A_{2}=A_{3}=0.355$. A quantitative comparison with [4] is proposed in Sec. III B 5, where we choose the setting $A_{1}=A_{2}=A_{3}=0.355$, and the impact of changing the eddy-damping constants is briefly illustrated and discussed in the Appendix.

\section{DYNAMICS AT VERY LARGE REYNOLDS NUMBERS}

In this section the present anisotropic EDQNM modeling, extended to active scalar dynamics, is used to investigate the large-Reynolds-number regimes of unstably stratified turbulence at $\mathrm{Sc}=1$. This part should be considered as a validation of our approach since the main features of [5] are recovered. In addition, throughout this section, qualitative comparisons with the cases of passive scalar dynamics (HITSG) and shear flows are presented since these two configurations were addressed with the same closure previously $[13,14]$.

First, the inertial scaling of the kinetic energy, scalar variance, and scalar flux spectra are addressed, along with some considerations about the large-scale initial conditions $\sigma$ and $\sigma_{T}$. Then the time evolution of one-point statistics is studied, such as the kinetic energy and its exponential growth rate, the Froude number, the mixing parameter, and global anisotropy indicators. The influence of large-scale initial conditions $\sigma$ and of the intensity of the stratification $N$ on the asymptotic anisotropic states of the previous quantities is also analyzed. Afterward, a quantitative comparison with the axisymmetric EDQNM model is proposed. Finally, the scale-by-scale repartition of anisotropy in spectral space is addressed and some considerations of the structure of the flow are proposed. 


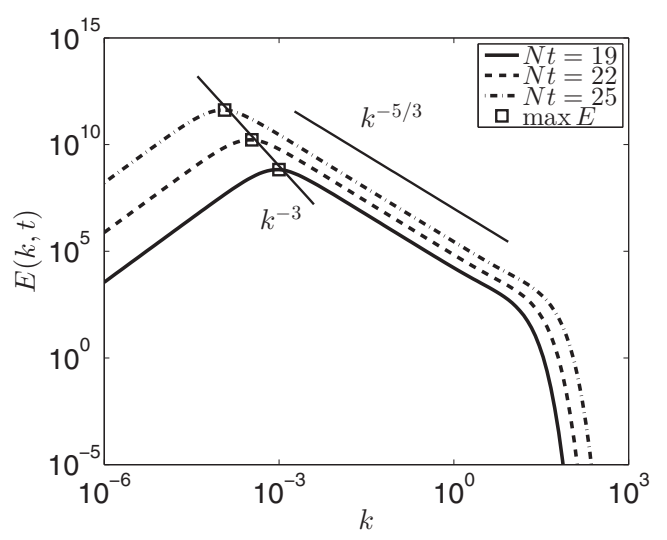

(a)

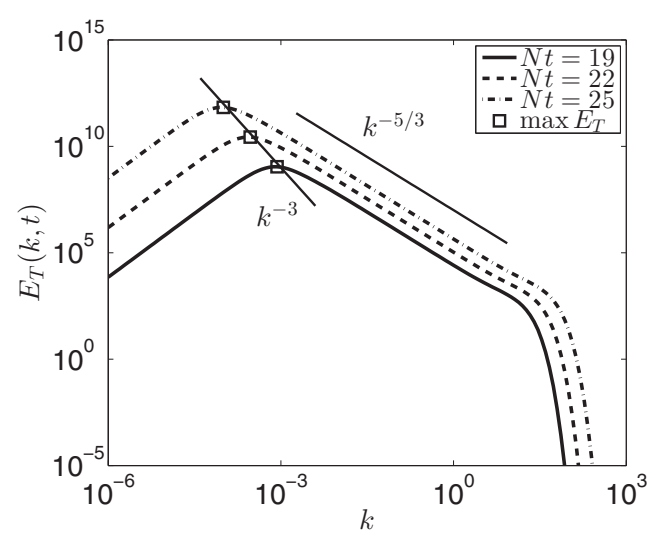

(b)

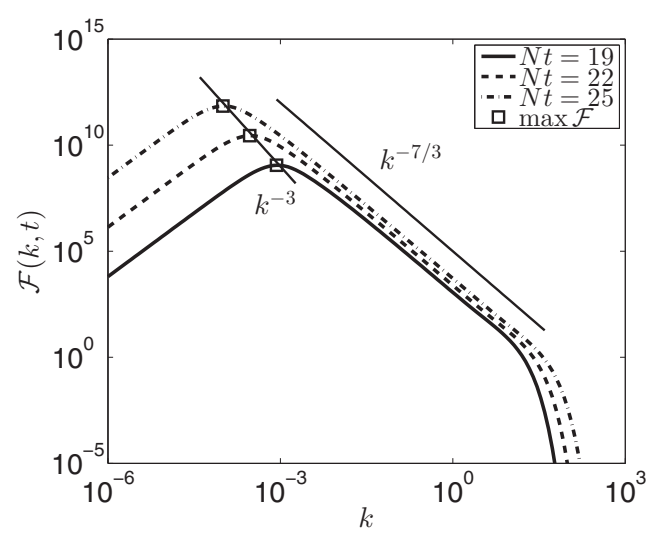

(c)

FIG. 2. Evolution of the spectra in Saffman USHT: (a) kinetic energy spectrum $E(k, t)$, (b) scalar variance spectrum $E_{T}(k, t)$, and (c) cospectrum $\mathcal{F}(k, t)$. Squares denote the peaks of the spectra, which are represented at three dimensionless times $N t=19,22$, and 25 and $\operatorname{Re}_{\lambda}(N t=25)=3 \times 10^{4}$.

\section{A. Spectral scaling and infrared dynamics}

In this section the emphasis is put on the three main spectra of USHT: the kinetic energy spectrum $E(k, t)$, the scalar variance spectrum $E_{T}(k, t)$, and the cospectrum $\mathcal{F}(k, t)$, which are investigated at large Reynolds numbers. First, the inertial scaling is discussed and then we focus on the infrared dynamics.

\section{Inertial scaling and compensated spectra}

First, the scaling of $E(k, t), E_{T}(k, t)$, and $\mathcal{F}(k, t)$ is studied at large Reynolds numbers. Similarly to the passive scalar case of HITSG, both $E$ and $E_{T}$ scale in $k^{-5 / 3}$ in the inertial range and $\mathcal{F}$ in $k^{-7 / 3}$, as revealed in Fig. 2.

Moreover, we note from Fig. 2 that the peaks of the three spectra evolve in $k^{-3}$ with increasing $N t$, as already assessed in Fig. 11 of [4]. We propose here a brief argument, which is inspired by a Rayleigh-Taylor analysis in Ref. [29]. Let us call $E_{\max }$ the maximum of the kinetic energy spectrum, which represents the kinetic energy $K$ at the integral scale $L$, so that $E_{\max }(t) \sim L(t) K(t)$. At large Reynolds numbers, in the self-similar state, the kinetic energy grows exponentially at the rate $\beta$ [4], so $K \sim \exp (\beta N t)$. Furthermore, by dimensional analysis, one has $L \sim K^{3 / 2} / \epsilon$, where the kinetic 


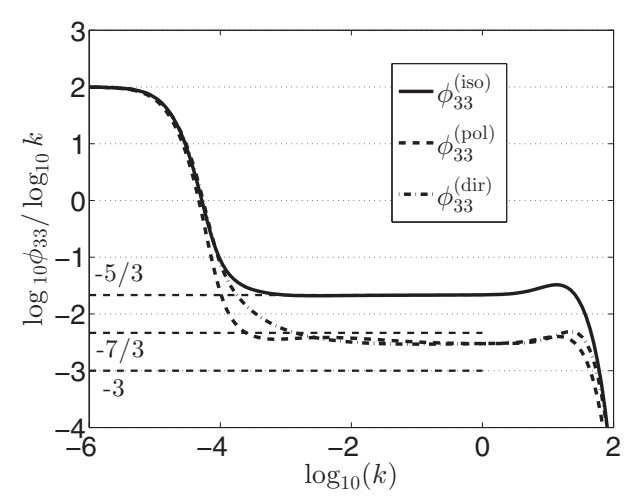

(a)

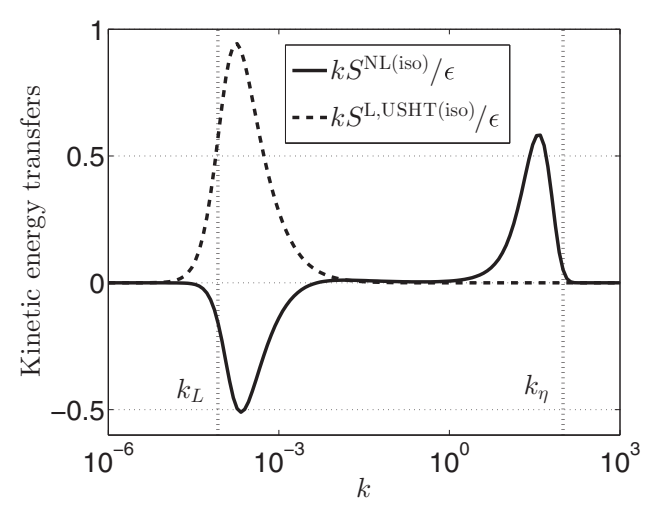

(b)

FIG. 3. (a) Spectral slope of the isotropic, directional, and polarization parts of the spectral tensor $\phi_{33}(k, t)$, with $\log _{10} \phi_{33} / \log _{10} k$ as a function of $\log _{10} k$. (b) Normalized linear and nonlinear transfers of kinetic energy. Both are for Saffman USHT at $N t=25$, where $\operatorname{Re}_{\lambda}=3 \times 10^{4}$.

energy dissipation rate grows exponentially at the rate $\beta$ as well, so $L \sim \exp (\beta N t / 2)$. This eventually gives $E_{\max } \sim \exp (3 \beta N t / 2)$. Then, at high Reynolds numbers, one can assume that $k_{\max }(t) \sim k_{L}(t)$, so the time $t$ can be expressed as follows: $t \sim-2 \ln \left(k_{L}\right) /(\beta N)$. Injecting this formula into $E_{\max }$ finally provides

$$
E_{\max }(t) \sim k_{L}^{-3},
$$

in agreement with numerical simulations.

Furthermore, the possibility of an anisotropic correction to the Kolmogorov spectra is discussed in Ref. [5], where it is shown numerically that the anisotropic part of $\phi_{33}(k, t)$ scales in $k^{-3}$ (mostly at the beginning of the inertial range). Nevertheless, in the corresponding Fig. 12 of [5], it appears that $k^{-3}$ is steeper than the inertial range slope obtained numerically. This is in agreement with our numerical simulations displayed in Fig. 3(a), where the isotropic, directional, and polarization parts of $\phi_{33}(k, t)$ are presented. Clearly, the isotropic part $\phi_{33}^{\text {(iso) }}$ scales in $k^{-5 / 3}$, whereas one has for $\phi_{33}^{\text {(pol) }}$ (and $\phi_{33}^{(\mathrm{dir})}$ ) a spectral slope steeper than $k^{-7 / 3}$, but not as sharp as $k^{-3}$. Our results are qualitatively in good agreement with the recent DNS [6], where the spectral scaling of $\phi_{33}^{(\mathrm{pol})}$ is also closer to $k^{-7 / 3}$ than $k^{-3}$. Furthermore, it is recovered, in agreement with [5], that the polarization part is more intense at large scales than the directional one.

In Fig. 3(b) the linear and nonlinear transfers of kinetic energy are displayed for Saffman turbulence. It is revealed that at large scales, linear production mechanisms dominate over nonlinear transfers that take energy from these large scales and bring it to smaller scales. This feature, that production is strong at large scales and thus that anisotropy is dominant at large scales, will be used later on.

Now we investigate the inertial scaling of $E(k, t), E_{T}(k, t)$, and $\mathcal{F}(k, t)$. Basically, we saw in Figs. 2 and 3(a) that both $E$ and $E_{T}$ scale in $k^{-5 / 3}$ in the inertial range and that $\mathcal{F}$ scales in $k^{-7 / 3}$. The remaining point to study is the value of the Kolmogorov and Corrsin-Obukhov constants $K_{0}$ and $K_{\mathrm{CO}}$, respectively. These constants are obtained by compensating the spectra with an adapted scaling. For the kinetic energy and buoyancy spectra, it is shown in Fig. 4(a) that the usual isotropic inertial scalings

$$
\begin{gathered}
E_{\text {inertial }}(k, t)=E(k, t) \epsilon^{-2 / 3} k^{5 / 3}, \\
E_{\mathrm{T}, \text { inertial }}(k, t)=E_{T}(k, t) \epsilon_{T}^{-1} \epsilon^{1 / 3} k^{5 / 3}
\end{gathered}
$$




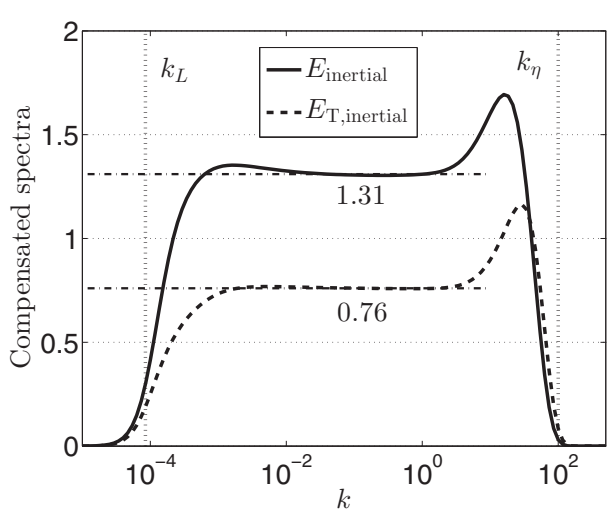

(a)

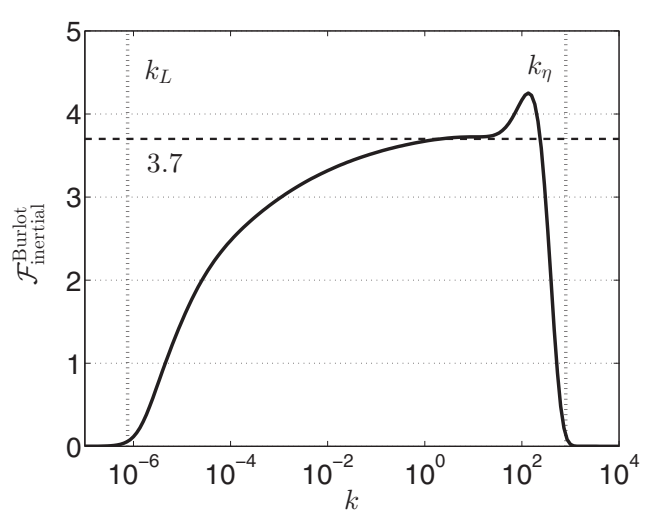

(b)

FIG. 4. Compensated spectra in the inertial range, with the integral and Kolmogorov wave numbers $k_{L}$ and $k_{\eta}$ : (a) compensated kinetic energy spectrum (45) and scalar variance spectrum (46) for $\sigma=2$ at $\operatorname{Re}_{\lambda}(N t=$ $25)=3 \times 10^{4}$, along with the Kolmogorov constant $K_{0}=1.31$ and Corrsin-Obukhov constant $K_{\mathrm{CO}}=0.76$, and (b) compensated cospectrum with the scaling (48) for $\sigma=1$ at $\operatorname{Re}_{\lambda}(N t=30)=3 \times 10^{6}$. The Lumley scaling (47) is not displayed since the curve is similar, but the plateau is located around 12 .

are relevant and allow one to recover classical values for the constants $K_{0}=1.31$ and $K_{\mathrm{CO}}=0.76$, similar to what is obtain for passive scalar dynamics [14,24]. This is different when it comes to the cospectrum $\mathcal{F}$. First, let us point out that there exist two different inertial scalings: the classical one proposed by Lumley [30], which was shown to work nicely for passive scalar dynamics in HITSG [14], with a constant $C_{\mathcal{F}} \simeq 3$, and a more recent one, which can be found in Ref. [5], which, unlike Lumley's, takes into account the scalar dissipation rate $\epsilon_{T}$,

$$
\begin{gathered}
\mathcal{F}_{\text {inertial }}^{\text {Lumley }}(k, t)=\mathcal{F}(k, t) N^{-1} \epsilon^{-1 / 3} k^{7 / 3}, \\
\mathcal{F}_{\text {inertial }}^{\text {Burlot }}(k, t)=\mathcal{F}(k, t) N^{-1}\left(K_{0} \epsilon^{1 / 3}+K_{\mathrm{CO}} \epsilon_{T} \epsilon^{-2 / 3}\right)^{-1} k^{7 / 3} .
\end{gathered}
$$

The inertial scaling $\mathcal{F}_{\text {inertial }}^{\text {Burlot }}$ was first derived in Ref. [2] and inspired by [31]. Taking into account the scalar dissipation rate $\epsilon_{T}$ in the scaling seems a priori relevant. Indeed, unlike passive scalar dynamics where $\mathcal{F}$ can be determined numerically without $E_{T}$, the buoyant field is critical in the dynamics of USHT and acts as a production term for the cospectrum. This scaling $\mathcal{F}_{\text {inertial }}^{\text {Burlot }}$ is presented in Fig. 4(b) at the very large Reynolds number $\operatorname{Re}_{\lambda} \simeq 3 \times 10^{6}$, where the plateau of the compensated cospectrum starts appearing around 3.7, whereas for the passive scalar case, at lower Reynolds numbers $10^{4} \leqslant \operatorname{Re}_{\lambda} \leqslant 10^{5}$, the compensated $\mathcal{F}$ was displaying a clear plateau around $C_{\mathcal{F}} \simeq 3$ in the inertial range [14]. Nevertheless, the scaling $\mathcal{F}_{\text {inertial }}^{\text {Burlot }}$ seems to be more relevant than Lumley's. Indeed, for $\mathcal{F}_{\text {inertial }}^{\text {Burlo }}$, a plateau appears around 3.7, whereas $\mathcal{F}_{\text {inertial }}^{\text {Lumley }}$ settles around 12 and is consequently not displayed in Fig. 4(b), which is much higher than values of usual constants close to unity such as the Kolmogorov and Corrsin-Obukhov ones. The fact the scaling (48) is better than $\mathcal{F}_{\text {inertial }}^{\text {Lumley }}$ is very likely because it takes the scalar dissipation rate $\epsilon_{T}$ into account.

\section{Infrared dynamics: Large-scale initial conditions}

Now that the inertial range of the spectra has been investigated, the infrared dynamics is considered. It is recalled that in HITSG for a passive scalar, if initially $\sigma_{T}$ is different from $\sigma$ (greater or lesser, it does not matter), it always results in $\sigma_{T}=\sigma$ after a few turnover times (see Appendix A of [14]). In USHT, it is fundamentally different since because of stratification, all of the three main spectra $E, E_{T}$, and $\mathcal{F}$ are coupled through the linear production terms at large scales. Hence, the most energetic initial spectrum, the one with the smallest infrared slope, imposes the minimum of 


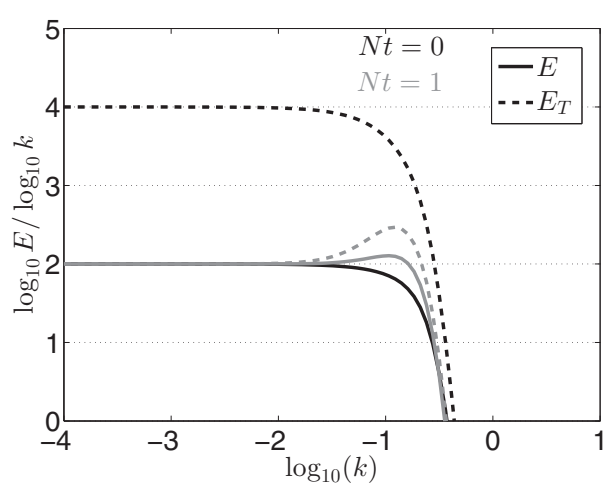

(a)

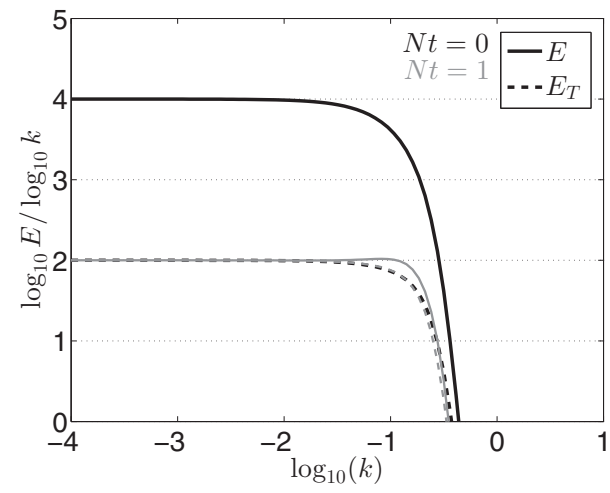

(b)

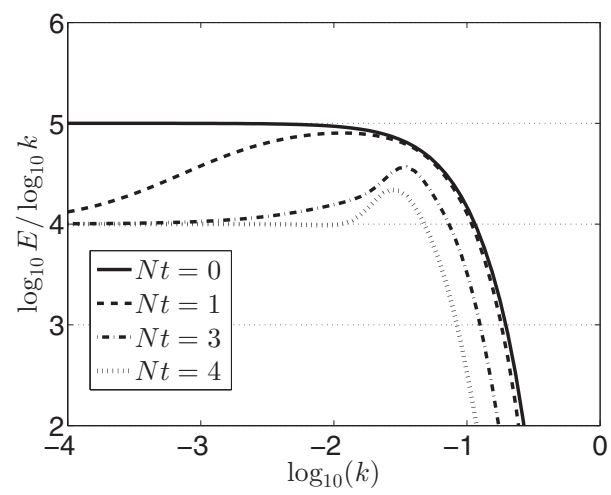

(c)

FIG. 5. Infrared dynamics of $E(k, t)$ and $E_{T}(k, t)$, with $\log _{10} E / \log _{10} k$ as a function of $\log _{10} k:$ (a) $\sigma(t=$ $0)=2$ and $\sigma_{T}(t=0)=4$, with black curves at $N t=0$ and gray ones at $N t=1$; (b) $\sigma(t=0)=4$ and $\sigma_{T}(t=0)=2$, with black curves at $N t=0$ and gray ones at $N t=1$; and (c) initially $\sigma(t=0)=5$ and then $\sigma$ varies from $N t=0$ to $N t=4$.

energy to the others, so that in the end one has always $\sigma=\sigma_{T}=\min \left[\sigma(t=0), \sigma_{T}(t=0)\right]$. This is completely different from HITSG where the passive scalar field has no effect on the kinetic field. Two examples are presented in Figs. 5(a) and 5(b). In Fig. 5(a) one has $\sigma(t=0)=2$ and $\sigma_{T}(t=0)=4$. The minimum energy is thus imposed by $E$ so that the scalar infrared slope results very rapidly, within one dimensionless time $N t$, in $\sigma_{T}=2$. This is identical to what happens in HITSG. In the opposite case illustrated in Fig. 5(b), one has $\sigma(t=0)=4$ and $\sigma_{T}(t=0)=2$ : The minimum energy is this time imposed by $E_{T}$ so that after one $N t, \sigma=2$. This case is completely different from HITSG, where $\sigma_{T}$ would have changed to 4 .

A last aspect is presented in Fig. 5(c): Because of strong backscatter of energy towards large scales when $\sigma \geqslant 4$, if the slope is initially $\sigma=5$, it eventually becomes $\sigma=4$ in a few dimensionless times $N t$. The same mechanism of strong inverse transfers of energy occurs in decaying isotropic turbulence [32].

From this analysis, one can choose, without any loss of generality, $\sigma=\sigma_{T} \leqslant 4$. This result for USHT infrared dynamics notably simplifies the study of asymptotic anisotropic states of the flow in Sec. III B. In particular, we choose to investigate only integer values of the infrared slopes: $\sigma=\sigma_{T}=\{1 ; 2 ; 3 ; 4\}$. 


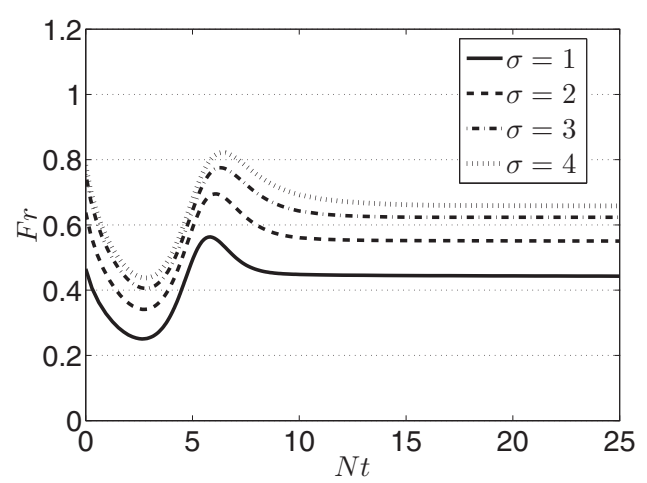

(a)

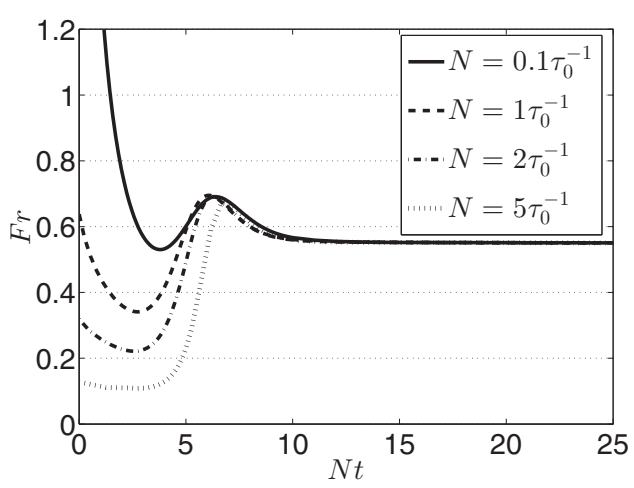

(b)

FIG. 6. Influence of initial parameters $\sigma$ and $N$ on the Froude number $\operatorname{Fr}=\epsilon / K N$ : (a) various $\sigma$ at $N=1 \tau_{0}^{-1}$ and (b) various $N$ for $\sigma=2$.

\section{B. One-point statistics}

Here two important quantities of unstably stratified turbulence are addressed, namely, the Froude number

$$
\operatorname{Fr}(t)=\frac{\epsilon(t)}{K(t) N}
$$

which is the ratio of the stratification characteristic time $1 / N$ to the inertial one $K / \epsilon$, and the mixing parameter

$$
\Lambda(t)=\frac{K_{T}(t)}{K(t)}
$$

which is the ratio of the scalar variance to kinetic energy. Since the scalar field is scaled as a buoyant velocity, $\Lambda$ is dimensionless. Then the exponential growth rate $\beta$ of the kinetic energy $K=\left\langle u_{i} u_{i}\right\rangle / 2$ is analyzed, along with the time evolution of global anisotropy using the normalized deviatoric Reynolds stress tensor $b_{i j}$. More precisely, the influence of $N$ and $\sigma$ on the asymptotic values reached by $\mathrm{Fr}, \Lambda, \beta$, and $b_{33}$ at large $N t$ and $\operatorname{Re}_{\lambda}$ is studied. Finally, a quantitative comparison with the axisymmetric EDQNM model [5] is proposed.

\section{Froude number Fr}

As said earlier, the Froude number Fr can be interpreted as the ratio of characteristic time scales of USHT. As such, it could be qualitatively compared to the shear rapidity $S_{R}=\epsilon / K S$ in shear flows, notably addressed with the same anisotropic EDQNM modeling in Ref. [13]. In Fig. 6(a) it is revealed that Fr, unlike $S_{R}$, depends on $\sigma$ in the asymptotic anisotropic state. Final values of Fr span from 0.44 for $\sigma=1$ to 0.66 for $\sigma=4$. The smaller $\sigma$ is, the smaller Fr becomes. This is expected since for small $\sigma$, large scales contain more energy and consequently are more anisotropic, because of the production terms that act dominantly at large scales, as illustrated in Fig. 3(b). With the production terms being dynamically dominant with a small $\sigma$, the characteristic time scale $1 / N$ diminishes, thus making Fr decrease. Nevertheless, the values reached here by Fr are slightly higher than the values obtained in Ref. [5] $(\mathrm{Fr}=0.3$ for $\sigma=1)$. This means that the flow within the present EDQNM modeling is less anisotropic than with the axisymmetric EDQNM model. It is shown in the Appendix that by changing the eddy-damping constants, we can increase the impact of stratification and thus reduce the Froude number. Asymptotic values of $\mathrm{Fr}$ are gathered in Table I.

In Fig. 6(b), however, it is shown that Fr, similarly to $S_{R}$, does not depend on the intensity of the mean gradient $N$, except at small $N t$ in the transitory regime. The larger $N$ is, the smaller Fr 
TABLE I. Comparison of the asymptotic values at large $\operatorname{Re}_{\lambda}$ of one-point statistics, obtained with the present anisotropic EDQNM modeling, and obtained with the axisymmetric EDQNM model [5]. For the present modeling, values in parentheses correspond to the setting of eddy-damping constants (EDC2) (see the Appendix for details).

\begin{tabular}{|c|c|c|c|c|c|c|c|c|}
\hline \multirow[b]{2}{*}{ Infrared slope $\sigma$} & \multicolumn{2}{|c|}{ Froude number Fr } & \multicolumn{2}{|c|}{ Mixing intensity $\Lambda$} & \multicolumn{2}{|c|}{ Growth rate $\beta$} & \multicolumn{2}{|c|}{ Global anisotropy $b_{33}$} \\
\hline & Present & Ref. [5] & Present & Ref. [5] & Present & Ref. [5] & Present & Ref. [5] \\
\hline 1 & $0.443(0.265)$ & 0.306 & $1.607(1.580)$ & 1.56 & 0.893 & 1 & $0.265(0.287)$ & 0.410 \\
\hline 2 & $0.551(0.337)$ & 0.385 & $1.508(1.466)$ & 1.45 & 0.715 & $4 / 5$ & $0.239(0.260)$ & 0.375 \\
\hline 3 & $0.624(0.387)$ & 0.435 & $1.440(1.387)$ & 1.37 & 0.596 & $2 / 3$ & $0.224(0.242)$ & 0.346 \\
\hline 4 & $0.659(0.412)$ & 0.460 & 1.407 (1.347) & 1.31 & 0.540 & $4 / 7$ & $0.217(0.234)$ & 0.323 \\
\hline
\end{tabular}

is initially. This is consistent with a strong stratification intensity making production of buoyant fluctuations dominant initially.

\section{Mixing intensity $\Lambda$}

Now the mixing intensity $\Lambda$ is addressed. Similarly to Fr, its asymptotic value depends on $\sigma$ as recovered in Fig. 7(a), but not on its initial value $\Lambda(0)$, except for short times of course as shown in Fig. 7(b). The dependence on $N$ is not presented since it is very similar to what happens for Fr, i.e., a dependence on $N$ only at short times. The final values of $\Lambda$ are contained between 1.6 for $\sigma=1$ and 1.4 for $\sigma=4$, which is quantitatively in good agreement with [5]. Asymptotic values of $\Lambda$ are gathered in Table I.

Let us point out that the initial variations of $K_{T} / K$ at a fixed $\sigma$ strongly depend on parameters such as the initial Reynolds number $\operatorname{Re}_{\lambda}(0)$ and the stratification frequency $N$. Indeed, $\Lambda$ initially decreases in Ref. [5], whereas it initially increases here. This is because of a different initial Reynolds number. Here $\operatorname{Re}_{\lambda}(0) \simeq 5$ implies that linear production mechanisms dominate whatever the value of $N$ is roughly $N \geqslant 0.1 \tau_{0}^{-1}$. In Ref. [5], $\operatorname{Re}_{\lambda}(0) \simeq 70$, which requires at least $N=1 \tau_{0}^{-1}$ to make $\Lambda$ increase initially, meaning that linear production overcomes nonlinearity. This is illustrated in Fig. 7(c).

\section{Exponential growth rate $\beta$}

The emphasis is now put on the exponential growth rate $\beta$ of the kinetic energy $K(t)$ in USHT. It was assessed by Burlot and co-workers that this growth rate strongly depends on the large-scale

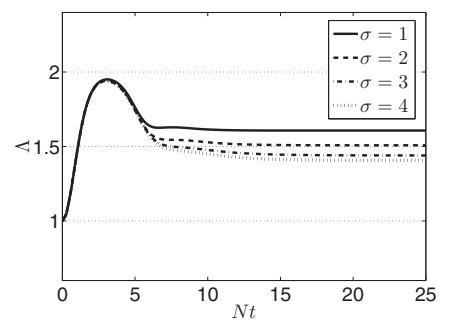

(a)

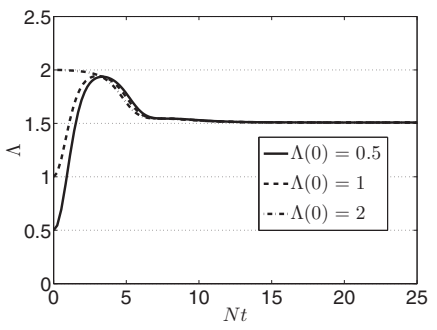

(b)

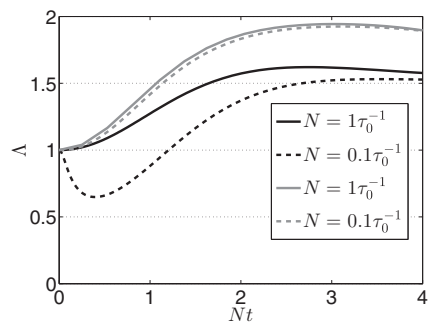

(c)

FIG. 7. Mixing intensity $\Lambda$ for different configurations: (a) various $\sigma$ at $N=1 \tau_{0}^{-1}$, (b) various initial values $\Lambda(0)$ for $\sigma=2$ and $N=1 \tau_{0}^{-1}$, and (c) short time dynamics of $\Lambda$ for various $N$ and $\operatorname{Re}_{\lambda}(0)$ with $\sigma=2$. Here $\operatorname{Re}_{\lambda}(0)=70$ is shown in black and $\operatorname{Re}_{\lambda}(0)=5$ in gray. 
TABLE II. Comparison between the present theoretical prediction $\beta_{\mathrm{th}}$ for the kinetic energy exponential growth rate and the numerical result $\beta$ obtained with our anisotropic EDQNM modeling. The prediction $\beta_{\text {Burlot }}$ is reported as well.

\begin{tabular}{lccc}
\hline \hline $\begin{array}{l}\text { Large-scale } \\
\text { initial condition } \sigma\end{array}$ & $\begin{array}{c}\text { Present } \\
\text { prediction } \beta_{\text {th }}\end{array}$ & $\begin{array}{c}\text { Present } \\
\text { EDQNM model } \beta\end{array}$ & $\begin{array}{c}\text { Burlot } \text { et al. } \\
\text { prediction } \beta_{\text {Burlot }}\end{array}$ \\
\hline 1 & 0.894 & 0.893 & 1 \\
2 & 0.716 & 0.715 & $4 / 5$ \\
3 & 0.596 & 0.596 & $2 / 3$ \\
4 & 0.511 & 0.540 & $4 / 7$ \\
\hline \hline
\end{tabular}

initial condition $\sigma$, according to the theoretical prediction

$$
K(t) \sim K(0) \exp \left(\beta_{\text {Burlot }} N t\right), \quad \beta_{\text {Burlot }}=\frac{4}{\sigma+3},
$$

which comes from the more general work [3] where the stratification frequency $N$ can vary. Equation (51) corresponds to the specific case where $N$ is constant. Furthermore, (51) relies on the fact that the largest eigenvalue of the linear operator of the evolution equations of [4] is $2 N$. Indeed, equalizing the growth rate of their linear limit $E \sim \exp (2 N t)$ with the one coming from dimensional analysis $E \sim \exp [(\sigma+3) \beta N t / 2]$ directly yields (51).

In our case, because of the anisotropy modeling through a truncated expansion into spherical harmonics, our evolution equations (28)-(33) are different from those of the axisymmetric EDQNM model. The largest eigenvalue of the linear operator associated with (28)-(33) is $4 N / \sqrt{5}$ here, in contrast to $2 \mathrm{~N}$ from [4-6]. This leads to a different theoretical prediction for the exponential growth rate of the kinetic energy

$$
K(t) \sim K(0) \exp \left(\beta_{\mathrm{th}} N t\right), \quad \beta_{\mathrm{th}}=\frac{8}{\sqrt{5}(\sigma+3)} .
$$

The present theoretical predictions and those of [4] are gathered in Table II. It is worth noting that for a given large-scale initial condition $\sigma$, our predictions yield a smaller growth rate than those of Burlot and co-workers. This is very likely due to our truncated expansion into spherical harmonics of the spectral correlations. Indeed, because of the truncation, there is a loss of angular information, so only partial anisotropy of the flow is captured: This is consistent with a final global anisotropy that is smaller here than in Ref. [4]. We could conjecture that taking into account more spherical harmonics would increase the exponential growth rate of the kinetic energy up to the limit $\beta_{\text {Burlot }}$.

The predictions for the exponential growth rate of the kinetic energy are compared to our numerical results in Fig. 8(a). The agreement is excellent: For $\sigma=1,2$, and 3, the prediction $\beta_{\text {th }}$ is recovered within $1 \%$. There is a slight difference in the case of Batchelor turbulence, where the numerical result is $5 \%$ higher than the prediction: This is very likely because of the strong inverse nonlinear transfers that naturally occur in Batchelor turbulence [32]. One could add a backscatter parameter, i.e., a correction for Batchelor turbulence, to the prediction (52) in the specific case $\sigma=4$, as usually done for decaying HIT [33,34]. In Batchelor HIT, one usually replaces $\sigma$ by $\sigma-p$, where $p=0.55$ is the backscatter parameter. Here, for USHT, in order to adapt the theoretical prediction in Batchelor turbulence to our numerical result, a least-squares fit leads to the backscatter parameter $p_{\text {USHT }}=0.37$, so

$$
\beta_{\mathrm{th}}=\frac{8}{\sqrt{5}\left(\sigma-p_{\mathrm{USHT}}+3\right)}, \quad p_{\mathrm{USHT}}= \begin{cases}0 & \text { for } \sigma \leqslant 3 \\ 0.37 & \text { for } \sigma=4 .\end{cases}
$$

It is revealed in Fig. 8(b) that the scalar variance $K_{T}$ and the mixed correlation $K_{\mathcal{F}}$ both grow at the same rate $\beta$, in agreement with [4-6]. This can be qualitatively compared with the case of a 


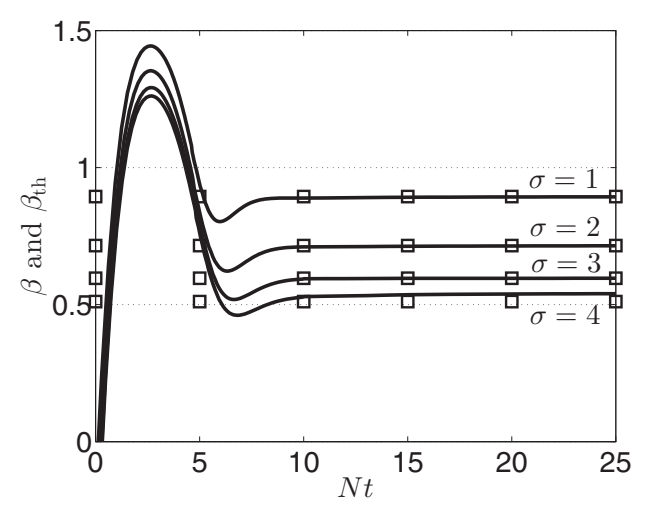

(a)

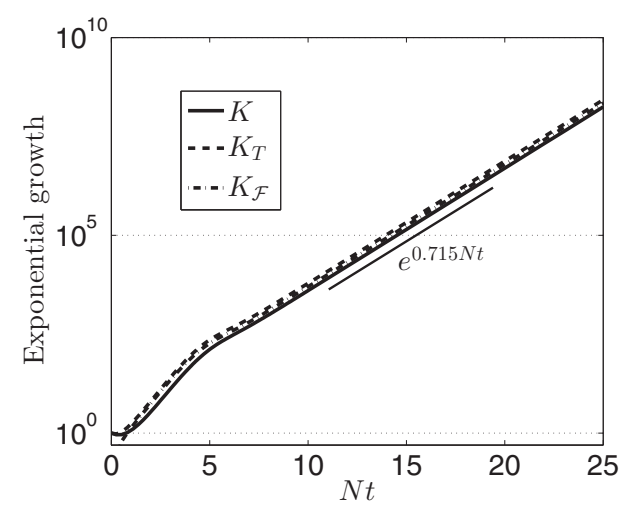

(b)

FIG. 8. (a) Kinetic energy exponential growth rate $\beta$ for $\sigma=1,2,3$, and 4. Straight lines indicate the numerical results and squares the theoretical prediction (52), without the correction $p_{\mathrm{USHT}}$. (b) Exponential growth of $K, K_{T}$, and $K_{\mathcal{F}}$ for $\sigma=2$.

passive scalar field advected by a turbulent shear flow with an imposed mean scalar gradient, where $K_{T}$ and $K_{\mathcal{F}}$ also grow exponentially with the same rate as $K$ [14].

In conclusion, the kinetic energy exponential growth rate strongly depends on the large-scale initial conditions $\sigma$ in the asymptotic states of USHT. This is interesting, since for shear flows, $K(t)$ is growing at the same rate independently of $\sigma[12,13]$, at least within the same anisotropic EDQNM modeling. This illustrates two intrinsically different mechanisms of kinetic energy production in shear flows and USHT.

\section{Global anisotropy $b_{33}$}

The time evolution of global anisotropy is now addressed. The scale-by-scale distribution of anisotropy is the subject of Sec. III C. First, the global anisotropy is investigated at the velocity level with the classical indicator $b_{i j}$, defined as the normalized deviatoric part of the Reynolds tensor

$$
b_{i j}=\frac{\left\langle u_{i} u_{j}\right\rangle}{2 K}-\frac{\delta_{i j}}{3}=b_{i j}^{(\mathrm{dir})}+b_{i j}^{(\mathrm{pol})}
$$

which can be split into two parts due to the decomposition of the spectral Reynolds tensor $\hat{R}_{i j}$ into polarization and directional anisotropies [11]. Therefore, $b_{i j}$ can be computed using $H_{i j}^{(\mathrm{pol})}$ and $H_{i j}^{(\mathrm{dir})}$, as done in Refs. [5,12-14], according to

$$
b_{i j}(t)=\frac{1}{K(t)} \int_{0}^{\infty} E(k, t)\left[H_{i j}^{(\mathrm{dir})}(k, t)+H_{i j}^{(\mathrm{pol})}(k, t)\right] d k .
$$

Similarly, one can define a scalar anisotropy indicator $b_{i j}^{T}$ as in Ref. [14], which only reflects directional anisotropy

$$
b_{i j}^{T}(t)=\frac{1}{K_{T}(t)} \int_{0}^{\infty} E_{T}(k, t) H_{i j}^{(T)}(k, t) d k .
$$

In Figs. 9(a)- 9(d), $b_{33}$ and $b_{33}^{T}$ first increase, which shows the departure from the isotropic state, and then decrease and reach a final nonzero value. This decrease is the signature of a return to isotropy of the small scales when the Reynolds number increases, and this will be explained in Sec. III C.

More specifically, it is revealed in Fig. 9(a) that polarization anisotropy for $b_{33}$ is stronger than the directional one, in agreement with [5]. The strong anisotropy in the component ( $)_{33}$ furthermore shows that turbulent structures mainly align with the mean scalar gradient. In addition, the values 


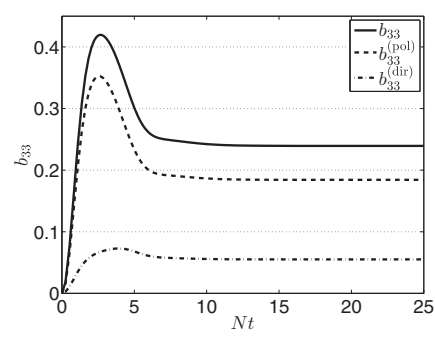

(a)

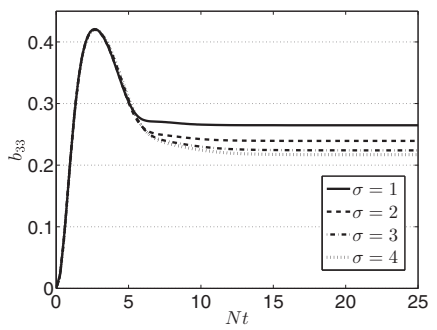

(b)

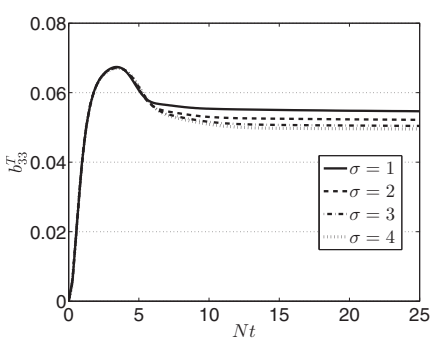

(c)

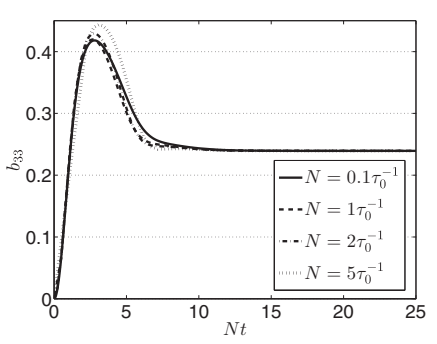

(d)

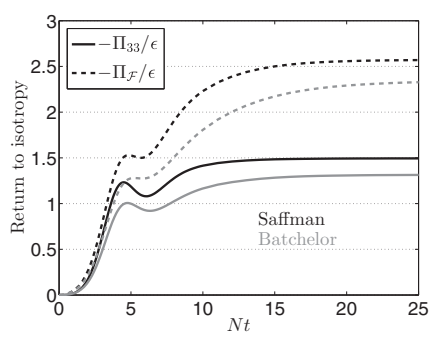

(e)

FIG. 9. Global anisotropy indicators for the velocity and scalar fields: (a) polarization and directional anisotropy parts of $b_{33}$ for $N=1 \tau_{0}^{-1}$ and $\sigma=2$, (b) $b_{33}$ at $N=1 \tau_{0}^{-1}$ for various $\sigma$, (c) $b_{33}^{T}$ at $N=1 \tau_{0}^{-1}$ for various $\sigma$, (d) $b_{33}$ at $\sigma=2$ for various $N$ (the same behavior is observed for $b_{33}^{T}$ ), and (e) a return to isotropy, with normalized pressure-velocity $\Pi_{33}$ and pressure-scalar $\Pi_{\mathcal{F}}$ correlations in Saffman (black) and Batchelor (gray) turbulence.

reached by $b_{33}^{T}$ in Fig. 9(c) are quite similar to the ones reached by $b_{33}^{(\mathrm{dir})}$. Another important feature is to compare the influence of initial conditions, such as $\sigma$ and $N$, on the final state of anisotropy. The same conclusions as for Fr and $\Lambda$ are drawn for $b_{33}$ and $b_{33}^{T}$ : As shown in Figs. 9(b)-9(d), varying $N$ affects only the short time dynamics of $b_{33}$ (the same is observed for $b_{33}^{T}$ ), whereas increasing $\sigma$ decreases the asymptotic values of the velocity and scalar anisotropy indicators. The latter feature is expected because by increasing $\sigma$, one diminishes the large-scale energy and consequently the amount of anisotropy injected in the flow.

The results obtained here are quite different from shear flows, where the asymptotic anisotropic state of $b_{i j}$ no longer depends on $\sigma$, whereas for both shear flows and USHT, varying the mean gradient intensity impacts only short times of $b_{i j}$. Hence, the main difference from [4,5] is that the present anisotropic EDQNM modeling underestimates the global anisotropy of the flow, so the asymptotic anisotropic states of $b_{33}$ are lower in our case. Values are reported in Table I. It is shown in the Appendix that we can slightly increase the global anisotropy of the flow by changing the eddy-damping constants.

Finally, in addition to these global anisotropy indicators, the pressure-velocity $\Pi_{33}$ and pressurescalar $\Pi_{\mathcal{F}}$ correlations are presented in Fig. 9(e); their definitions are, respectively,

$$
\begin{gathered}
\Pi_{\mathcal{F}}(t)=\left\langle p \frac{\partial c}{\partial x_{3}}\right\rangle=\int_{0}^{\infty} S_{3}^{\mathrm{F}, \mathrm{NL}}(k, t) d k \\
\Pi_{33}(t)=\left\langle 2 p \frac{\partial u_{3}}{\partial x_{3}}\right\rangle=2 \int_{0}^{\infty}\left(\frac{1}{3} S^{\mathrm{NL}(\mathrm{iso})}+S_{33}^{\mathrm{NL}(\mathrm{dir})}+S_{33}^{\mathrm{NL}(\mathrm{pol})}\right) d k .
\end{gathered}
$$

The return to isotropy at the level of the scalar flux is found to be more intense than the one of the velocity field. This is expected since the cospectrum is a purely anisotropic quantity, for which pressure is the destructive mechanism. Furthermore, in agreement with previous statements, the 


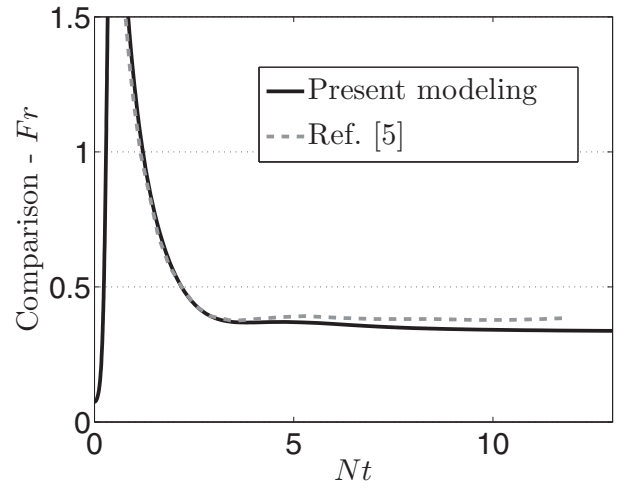

(a)

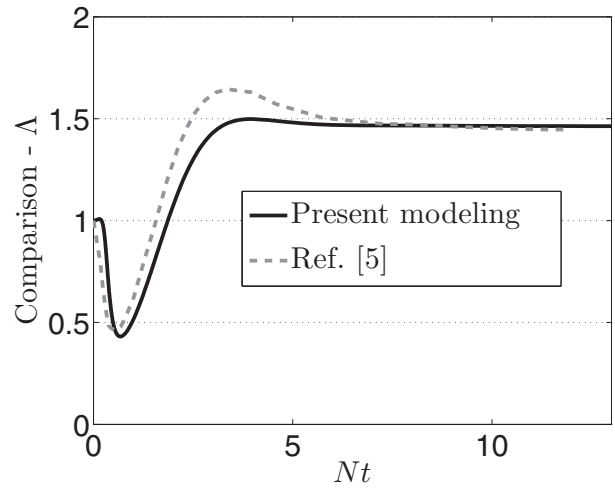

(b)

FIG. 10. Comparison of the present anisotropic EDQNM modeling with the axisymmetric EDQNM model [5] with $\sigma=2, \operatorname{Re}_{\lambda}(0) \simeq 70, N=4 \tau_{0}^{-1}$, and $k_{\text {peak }}=40 k_{L}(0)$ for (a) Froude number Fr and (b) mixing intensity $\Lambda$.

return to the isotropy mechanism is stronger for Saffman turbulence than for Batchelor turbulence, because large scales are less anisotropic in the latter case than in the former.

\section{Comparison with Refs. [4,5]}

In this section we compare quantitatively the results of our anisotropic EDQNM modeling to the axisymmetric EDQNM model [4,5], where the linear production terms are exactly treated, without any modeling of anisotropy. The emphasis is put on two one-point statistics investigated in the previous sections: the Froude number Fr, defined in Eq. (49), and the mixing intensity $\Lambda$ defined in Eq. (50).

First, it was observed in the previous sections that the asymptotic states reached with the present anisotropic EDQNM modeling are less anisotropic than the ones obtained in Ref. [5]; this was notably seen through Fr and $b_{33}$. In order to provide here a meaningful comparison, and only in this section, we use the eddy-damping constants $A_{1}=A_{2}=A_{3}=0.355$. The impact on USHT dynamics of the two different settings of eddy-damping constants is discussed in the Appendix: The setting $A_{1}=0.355, A_{2}=0$, and $A_{3}=1.3$ is called EDC1 for the present modeling, and the setting $A_{1}=A_{2}=A_{3}=0.355$ is called EDC2. So for the comparison, we use the setting EDC2 in Saffman turbulence $(\sigma=2)$, an initial turbulent Reynolds number close to $\operatorname{Re}_{T}=833$, with $\operatorname{Re}_{\lambda}=\sqrt{20 \operatorname{Re}_{T} / 3}$, and the initial peak of energy is $k_{\text {peak }}=40 k_{L}(0)$. Even though it is stated in Ref. [4] that the initial Froude number is $\mathrm{Fr}=1.2$, it seems to not be the case in Fig. 1(b) therein. Consequently, we choose the stratification frequency $N=4 \tau_{0}^{-1}$ so that the initial behavior of Fr is recovered; taking $1 \tau_{0}^{-1} \leqslant N \leqslant 4 \tau_{0}^{-1}$ would not have changed much.

The results are presented in Fig. 10. For the Froude number, the overall agreement is excellent: The transient regime is correctly captured and in particular the strong decrease; the asymptotic value of Fr is quite well recovered. For the mixing ratio $\Lambda$, the initial behavior is quite well captured, with very good agreement for the asymptotic value. In conclusion, there is satisfactory agreement with the axisymmetric EDQNM model if one changes the eddy-damping constants from EDC1 to EDC2. The drawback is that, as illustrated in the Appendix, by doing so the Corrsin-Obukhov constant decreases.

\section{Conclusion on one-point statistics}

In this section various one-point statistics of crucial importance in unstably stratified turbulence were studied: the Froude number Fr, the mixing intensity $\Lambda$, the growth rate $\beta$ of the kinetic energy, 


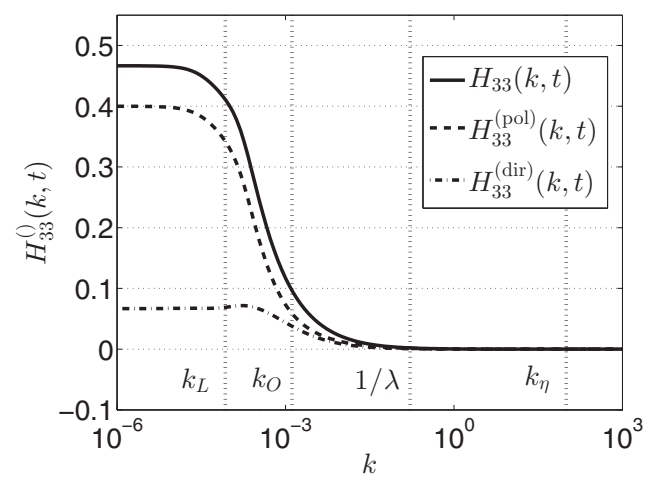

(a)

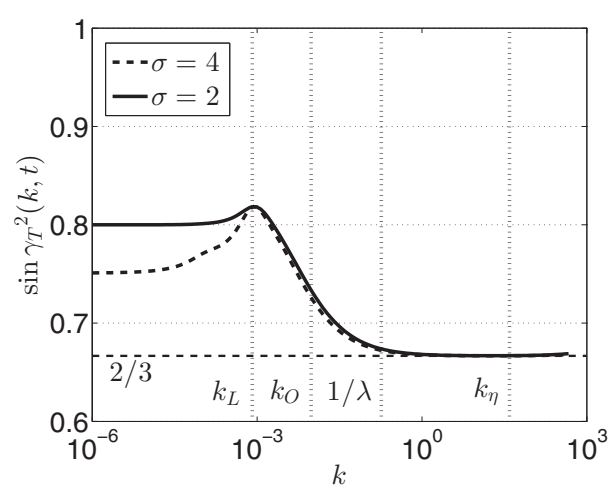

(b)

FIG. 11. Spectral anisotropy indicators, along with the integral, Ozmidov, and Kolmogorov wave numbers $k_{L}, k_{O}$, and $k_{\eta}$, respectively; the Taylor scale $\lambda$ is displayed as well: (a) $H_{33}^{(\mathrm{dir})}$ and $H_{33}^{(\mathrm{pol})}$ for $\sigma=2$ at $\operatorname{Re}{ }_{\lambda}(N t=$ $25)=3 \times 10^{4}$ and (b) $\sin ^{2}\left(\gamma_{T}\right)$ for $\sigma=2$ and $\sigma=4$ at different $N t$ so that for both $\operatorname{Re}_{\lambda}=3500$.

scalar variance, and scalar flux, and the velocity and scalar global anisotropy indicators $b_{33}$ and $b_{33}^{T}$. The different results, obtained in Refs. [4,5] and recovered here, could be summarized as follows.

(i) All these quantities strongly depend on $\sigma$ in the asymptotic anisotropic state at large $N t$ or equivalently at large Reynolds numbers. When $\sigma$ increases, the large-scale energy diminishes along with the quantity of anisotropy injected in the flow, so Fr increases and $\Lambda, \beta, b_{33}$, and $b_{33}^{T}$ decrease.

(ii) In contrast, the asymptotic anisotropic state does not depend on $N$ : Changing the intensity of the mean scalar gradient only impacts the short time dynamics.

It appears that our model can recover qualitatively all the features of USHT analyzed in Refs. [4-6]. Furthermore, good quantitative agreement is found. In addition, higher Reynolds numbers were reached here using a very reasonable amount of time and computational resources.

Finally, the main difference between the two approaches is that the flow is less anisotropic in our case, probably due to the truncation of the spherical harmonics expansion of spectral correlations for the modeling of anisotropy. The principal consequences are (i) an exponential growth rate of the kinetic energy $10 \%$ lower than in Ref. [4], but nevertheless in agreement with our theoretical prediction, and (ii) smaller values for $b_{33}$ as well. Asymptotic values of the one-point statistics analyzed so far, obtained with both the present anisotropic EDQNM modeling and the axisymmetric EDQNM model, are gathered in Table I.

Furthermore, throughout this section, qualitative comparisons were made with the cases of passive scalar dynamics (HITSG) and shear flows. It notably appeared that the asymptotic anisotropic states in USHT and shear flows strongly differ. Indeed, in shear flows, at least within the same anisotropic EDQNM modeling, the asymptotic anisotropic state does not depend on the mean-field gradient intensity or on the large-scale initial conditions $\sigma$.

\section{Scale-by-scale anisotropy and structure of the flow}

In this section the scale-by-scale distribution of anisotropy is investigated for the velocity and scalar fields, at the level of the second-order moments, with $H_{33}^{(\mathrm{dir})}(k, t), H_{33}^{(\mathrm{pol})}(k, t)$, and $H_{33}^{(T)}(k, t)$. More precisely, we use, instead of $H_{33}^{(T)}$ directly, the dimensionality parameter defined in Eq. (18), whose value is $2 / 3$ for isotropic scales.

It was shown in Figs. 9(a)-9(d) that in the asymptotic anisotropic state, the global anisotropy indicators $b_{33}$ and $b_{33}^{T}$ are nonzero, meaning that there is anisotropy in the flow. It is revealed in Fig. 11(a) that anisotropy is mainly gathered at large scales for the velocity field, as presented previously in Fig. 3(b), where $H_{33}^{(\mathrm{dir})}$ and $H_{33}^{(\mathrm{pol})}$ are different from zero, unlike small scales that have returned to 


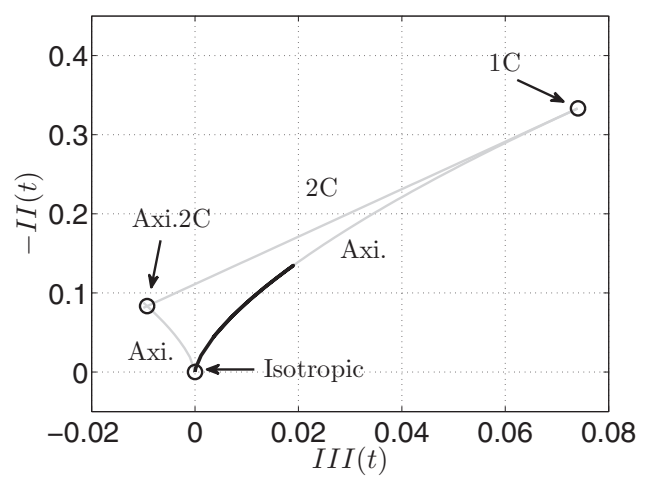

(a)

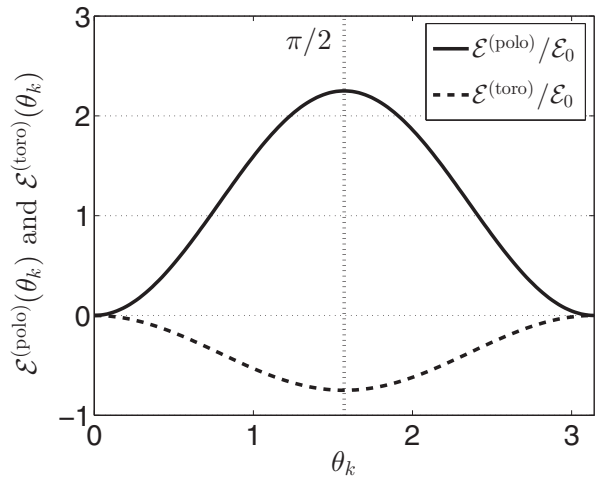

(b)

FIG. 12. (a) Lumley's triangle. Gray lines correspond to the boundaries between the isotropic twocomponent axisymmetric (Axi.2C) and one-component (1C) configurations and the black line corresponds to our simulation. (b) Normalized potentials $\mathcal{E}^{\text {(toro) }} / \mathcal{E}_{0}$ and $\mathcal{E}^{\text {(polo) }} / \mathcal{E}_{0}$, for $\sigma=2$, at a wave number located in the infrared range.

isotropy. One can remark that, as in Fig. 9(a), the polarization anisotropy is much stronger than the directional one at large scales. An interpretation of this is provided later. Similarly in Fig. 11(b) for the scalar field, small scales have returned to isotropy $\left(\sin ^{2} \gamma_{T}=2 / 3\right)$, whereas anisotropy is gathered at large scales. This figure additionally illustrates further that Saffman turbulence is globally more anisotropic than Batchelor turbulence, because the linear production at large scales is stronger in Saffman turbulence than in Batchelor turbulence. Interestingly, for both the velocity and scalar fields, Figs. 11(a) and 11(b) show that from the longitudinal Taylor scale $\lambda=\sqrt{20 \nu K / \epsilon}$, the scales have completely returned to isotropy. In particular, this indicates that even in USHT, isotropic statistics could be used for scales smaller than $\lambda$. The Ozmidov wave number $k_{O}=2 \pi \sqrt{N^{3} / \epsilon}$, defined in Ref. [6], is displayed as well: It is clear that for scales larger than $k_{O}$, stratification and anisotropy dominate, whereas for scales smaller, nonlinear transfers yield the return to isotropy mechanism.

In addition to the previous considerations about the distribution of anisotropy scale by scale, it is possible to obtain some qualitative information about the spatial structure of the flow. If one considers the Lumley triangle [35], which displays the second invariant of $b_{i j},-2 I I=b_{i j} b_{i j}$, as a function of the third one $3 I I I=b_{i j} b_{i l} b_{j l}$, one obtains the gray curves of Fig. 12(a). In our simulation (black curve), the flow evolves from an isotropic state toward a one-component state following an axisymmetric configuration and tends to be two dimensional, indicating an invariance along the direction of the mean scalar gradient (accordingly, the Reynolds stresses have a rodlike shape).

Another possibility is to investigate the energy contained in the toroidal and poloidal modes defined in Eq. (21) and linked to the spectral anisotropy descriptors $H_{33}^{\text {(dir) }}$ and $H_{33}^{(\mathrm{pol})}$ in Eqs. (26) and (27). The variations of both $\mathcal{E}^{\text {(toro) }}$ and $\mathcal{E}^{\text {(polo) }}$ as functions of $\theta_{k}$ are displayed in Fig. 12(b) for a wave number located in the infrared range: This is where the anisotropy is gathered, as shown in Fig. 11(a). For $0 \leqslant \theta_{k} \leqslant \pi$, one has $\mathcal{E}^{(\text {polo })}>\mathcal{E}^{(\text {toro) }}$ and the poloidal and toroidal potentials are maximum for $\theta_{k}=\pi / 2$. The poloidal mode being more intense means that in the configuration $\theta_{k}=\pi / 2$, spectral velocity fluctuations are preferentially aligned in the mean scalar gradient direction. This is an interesting feature. Indeed, in the previous sections it was underlined that polarization anisotropy is stronger than directional anisotropy at large scales. This is assessed in Fig. 12(b), where in the infrared range one has clearly $Z>\mathcal{E}^{\text {(dir) }}$. Consequently, the present results show that in USHT, a dominant polarization anisotropy corresponds to spectral velocity fluctuations mainly aligned with the mean scalar gradient, so the principal component is the poloidal one, in agreement with Fig. 12(a), where our simulation goes toward the one-component (1C) state. Also, for $\theta_{k}=0$ or $\theta_{k}=\pi$, in a plane perpendicular to the mean scalar gradient, $\mathcal{E}^{(\text {toro) }}=\mathcal{E}^{(\text {polo) }}$, so there is no polarization anisotropy. 


\section{PRESSURE SPECTRA AND HIGH SCHMIDT NUMBERS}

So far, the dynamics of USHT at $\mathrm{Sc}=1$ has been addressed at large Reynolds numbers and the strong dependence of the asymptotic anisotropic state on the infrared slope $\sigma$ has been recovered, with good overall agreement with the axisymmetric EDQNM model. In the present section, our anisotropic EDQNM modeling is applied to two cases. First, the pressure spectrum is studied and in particular its anisotropic part resulting from stratification, with a comparison to the pressure spectrum in shear flows. Then the case of very large Schmidt numbers Sc $\gg 1$, corresponding, for instance, to saltwater, is analyzed from a fundamental point of view, with the emphasis put on the scaling of the cospectrum.

\section{A. Pressure spectra in USHT and shear flows}

In this section pressure spectra in USHT are addressed. First, the spectral evolution equations of the pressure field are presented and then the anisotropic EDQNM modeling is used to evaluate both the isotropic and anisotropic parts of the pressure spectrum $E_{P}(k, t)$. The latter was notably investigated more than 30 years ago by George et al. [36] for shear flows. To do so, we additionally present the analytical calculations yielding the pressure spectra in shear flows since it was not done in Refs. [12,13].

\section{Modeling of pressure spectra in USHT and shear flows}

Here the equations leading to the pressure spectra in USHT are derived, i.e., the isotropic part that is always present and the anisotropic part arising because of the stratification. The anisotropic part of the pressure spectrum in shear flows is also presented.

The pressure fluctuations satisfy the Poisson equation obtained by taking the divergence of the Navier-Stokes equation (4),

$$
-\Delta p=\frac{\partial^{2} u_{i} u_{j}}{\partial x_{i} \partial x_{j}}+\lambda_{i} \frac{\partial c}{\partial x_{i}},
$$

where we keep $\lambda_{i}=\partial C / \partial x_{i}$ for generality and $C$ is the mean buoyant field. The spectral two-point second-order pressure correlation is defined as

$$
\mathcal{E}_{P}(\boldsymbol{k}, t) \delta(\boldsymbol{k}-\boldsymbol{p})=\left\langle\hat{p}(\boldsymbol{k}, t) \hat{p}^{*}(\boldsymbol{p}, t)\right\rangle,
$$

so the pressure spectrum reads

$$
E_{P}(k, t)=\int_{S_{k}} \mathcal{E}_{P}(\boldsymbol{k}, t) d^{2} \boldsymbol{k}
$$

Then, after some algebra, one gets

$$
\mathcal{E}_{P}(\boldsymbol{k}, t)=2 \alpha_{i} \alpha_{j} \alpha_{p} \alpha_{q} \int_{\boldsymbol{k}=\boldsymbol{p}+\boldsymbol{q}} \hat{R}_{i q}(\boldsymbol{p}, t) \hat{R}_{j p}(\boldsymbol{q}, t) d^{3} \boldsymbol{p}+\frac{\alpha_{i} \alpha_{j}}{k^{2}} \lambda_{i} \lambda_{j} \mathcal{E}^{T}(\boldsymbol{k}, t),
$$

where $\alpha_{i}=k_{i} / k$. The first contribution is purely isotropic and corresponds to the isotropic part of the pressure spectrum $E_{P}^{(\mathrm{iso})}$, also called the turbulence-turbulence interaction. A quasinormal procedure is used to compute this isotropic part of the pressure spectrum and the details of such calculations can be found either in Refs. [10,36], or more recently in Ref. [37]. The spherical average of the equation of $\mathcal{E}_{P}(\boldsymbol{k}, t)$ eventually gives

$$
E_{P}(k, t)=\underbrace{16 \pi^{2} \int_{\Delta_{k}} k p q\left(1-y^{2}\right)\left(1-z^{2}\right) \mathcal{E}_{0}^{\prime} \mathcal{E}_{0}^{\prime \prime} d p d q}_{E_{P}^{(\text {iso })}}+\underbrace{\frac{E_{T}}{k^{2}} \lambda_{i} \lambda_{j}\left(\frac{\delta_{i j}}{3}-2 H_{i j}^{(T)}\right)}_{E_{P}^{\text {(USHT) }}},
$$


where we call the second contribution $E_{P}^{(\mathrm{USHT})}$ the turbulence-unstable-stratification interaction, which involves the directional anisotropy of the scalar field $H_{i j}^{(T)}$. In addition, $\Delta_{k}$ is the domain where $k, p$, and $q$ are the lengths of the sides of the triangle formed by the triad $\boldsymbol{k}+\boldsymbol{p}+\boldsymbol{q}=\mathbf{0}$. Further, $x, y$, and $z$ are the cosines of the angles formed by $\boldsymbol{p}$ and $\boldsymbol{q}, \boldsymbol{q}$ and $\boldsymbol{k}$, and $\boldsymbol{k}$ and $\boldsymbol{p}$, respectively. The following compact notations are used: $\mathcal{E}_{0}^{\prime}=E(p) / 4 \pi p^{2}$ and $\mathcal{E}_{0}^{\prime \prime}=E(q) / 4 \pi q^{2}$.

The procedure for shear flows is very similar. The mean velocity gradient matrix is defined as $A_{i j}=\partial U_{i} / \partial x_{j}$, where $U_{i}$ is the mean velocity field, with $A_{13}=-S$, where $S$ is the shear intensity, as defined in Ref. [13]. In shear flows, the Poisson equation reads

$$
-\Delta p=\frac{\partial^{2} u_{i} u_{j}}{\partial x_{i} \partial x_{j}}+2 A_{i j} \frac{\partial u_{j}}{\partial x_{i}} \text {. }
$$

The Fourier transform yields

$$
\hat{p}(\boldsymbol{k}, t)=-\alpha_{i} \alpha_{j}{\widehat{u_{i} u_{j}}}_{j}(\boldsymbol{k}, t)+\frac{2 \mathrm{i}}{k} A_{i j} \alpha_{i} \hat{u}_{j}(\boldsymbol{k}, t),
$$

which provides, after some algebra very similar to what was used for USHT,

$$
\begin{aligned}
E_{P}(k, t)= & 16 \pi^{2} \int_{\Delta_{k}} k p q\left(1-y^{2}\right)\left(1-z^{2}\right) \mathcal{E}_{0}^{\prime} \mathcal{E}_{0}^{\prime \prime} d p d q \\
& +4 \frac{E}{k^{2}}\left\{\frac{1}{5} A_{i j}^{+} A_{i j}^{+}+\frac{1}{3} A_{i j}^{-} A_{i j}^{-}-H_{i l}^{(\mathrm{dir})}\left[\frac{6}{7} A_{i j}^{+} A_{j l}^{+}+2 A_{i j}^{-}\left(A_{l j}^{-}+2 A_{l j}^{+}\right)\right]\right\} \\
& +8 \frac{E}{k^{2}} H_{i l}^{(\mathrm{pol})}\left[\frac{3}{7} A_{i j}^{+} A_{l j}^{+}+A_{i j}^{-}\left(A_{l j}^{-}-\frac{2}{3} A_{l j}^{+}\right)\right],
\end{aligned}
$$

where $A_{i j}^{+}$and $A_{i j}^{-}$refer to the symmetric and antisymmetric parts of the mean velocity gradient matrix $A_{i j}$. The first contribution is $E_{P}^{(\text {iso) }}$ as before, whereas the second contribution $E_{P}^{(\mathrm{S})}$, called the turbulence-mean-shear interaction, arises from mean velocity gradients and involves the directional and polarization anisotropies of the velocity field.

\section{Numerical results for pressure spectra}

In this section pressure spectra obtained in USHT and in sustained shear flows are presented. For informative purposes, we begin with the pressure spectrum in shear flows, since it has been thoroughly investigated in Ref. [36]. Simulations of a previous work [13] are used.

Whatever framework is chosen, it directly follows from dimensional analysis that the isotropic part of the pressure spectrum, the turbulence-turbulence interaction, scales as

$$
E_{P}^{(\text {iso) }}(k, t)=C_{P} \epsilon^{4 / 3} k^{-7 / 3},
$$

where $C_{P}$ is a constant to be determined. The $k^{-7 / 3}$ scaling is assessed numerically in Fig. 13(a) and was previously verified in HIT [37,38] and shear flows [36]. Also, even if not presented, we recover the fact that the infrared slope of the isotropic pressure spectra is always $\sigma=2$, whatever the value imposed initially is, in agreement with [38]: $E_{P}^{\text {(iso) }}\left(k<k_{L}\right) \sim k^{2}$.

The compensated isotropic pressure spectra $E_{P}^{(\text {iso })} k^{7 / 3} \epsilon^{-4 / 3}$ for shear flows and USHT are presented in Figs. 13(b) and 14(b). The plateau settles around 2.5 for shear and 2.3 for USHT, which is quite close and proves some universality of the isotropic pressure spectrum between two completely different flows. Furthermore, these values are in good agreement with the prediction [36], where the constant would be $C_{P}=1.32 K_{0}^{2}=2.27$, close to our result for shear flows $C_{P}=2.5$.

The framework of a turbulent shear flow is now considered, where the turbulence-mean-shear interaction spectrum $E_{P}^{(\mathrm{S})}$ appears. Given its expression (66) with $E \sim k^{-5 / 3}$, it is straightforward 


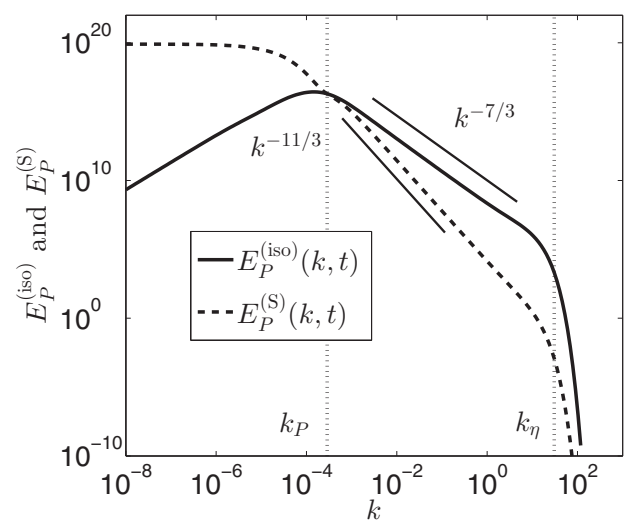

(a)

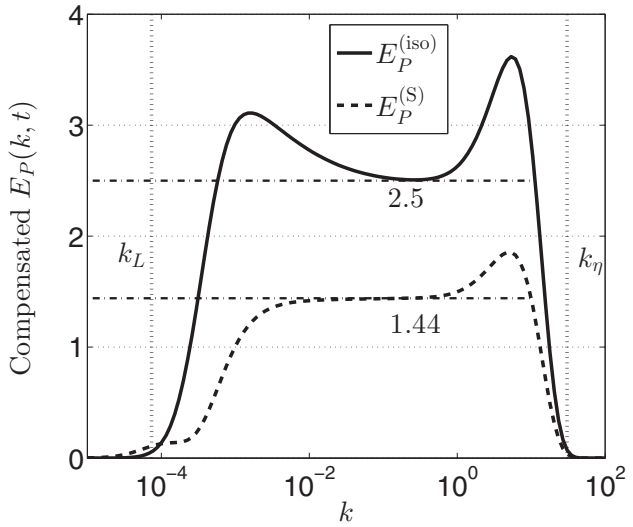

(b)

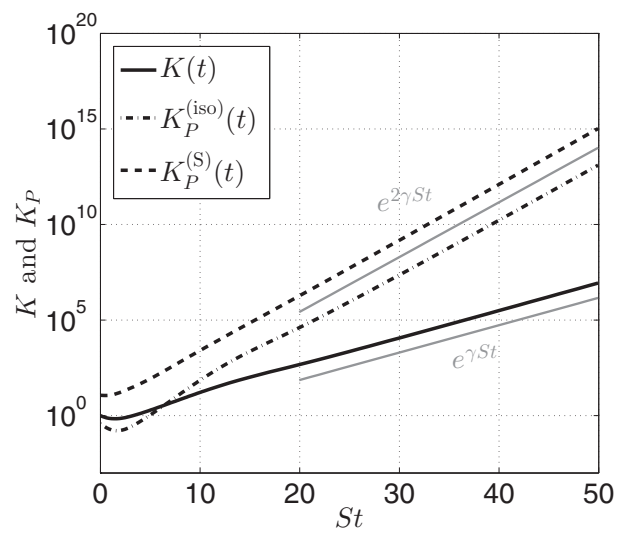

(c)

FIG. 13. Pressure spectra in shear flows for $\sigma=2$ at $\operatorname{Re}_{\lambda}=2 \times 10^{4}$ : (a) isotropic and anisotropic pressure spectra $E_{P}^{(\text {iso) }}$ and $E_{P}^{(\mathrm{S})}$, along with the Kolmogorov wave number $k_{\eta}$, at $\operatorname{Re}_{\lambda} \simeq 10^{4}$; (b) compensated pressure spectra $E_{P}^{(\text {iso) }} k^{7 / 3} \epsilon^{-4 / 3}$ and $E_{P}^{(\mathrm{S})} k^{11 / 3} \epsilon^{-2 / 3} / S^{2}$; and (c) isotropic and anisotropic parts $K_{P}^{\text {(iso) }}$ and $K_{P}^{(\mathrm{S})}$ of the pressure variance $K_{P}$, along with the kinetic energy $K$ for comparison. Here $\gamma$ is the exponential growth rate of the kinetic energy. The gray lines indicate the curves $\exp (\gamma S t)$ and $\exp (2 \gamma S t)$.

that it evolves in $k^{-11 / 3}$ in the inertial range and dimensional analysis yields

$$
E_{P}^{(\mathrm{S})}(k, t)=C_{P}^{(\mathrm{S})} S^{2} \epsilon^{2 / 3} k^{-11 / 3},
$$

as given in Ref. [36]. The $k^{-11 / 3}$ scaling is recovered in Fig. 13(a). The total pressure spectrum $E_{P}(k, t)$ evolves in $k^{-7 / 3}$ in the inertial range and is not presented since it cannot be distinguished from $E_{P}^{(\text {iso) }}$. In Fig. 13(b) the compensated $E_{P}^{(\mathrm{S})}$ indicates that $C_{P}^{(\mathrm{S})} \simeq 1.44$, which is close to the Kolmogorov constant, and this is expected since $E_{P}^{(\mathrm{S})}$ scales in $E / k^{2}$ in Eq. (66). Furthermore, the value $C_{P}^{(\mathrm{S})} \simeq 1.44$ is in good agreement with the prediction [36], where the constant would be $C_{P}^{(\mathrm{S})}=16 K_{0} / 15=1.40$.

The pressure variance, which can be divided into isotropic and anisotropic parts

$$
K_{P}(t)=\int_{0}^{\infty} E_{P}(k, t) d k=K_{P}^{(\mathrm{iso})}(t)+K_{P}^{(\mathrm{S})}(t),
$$




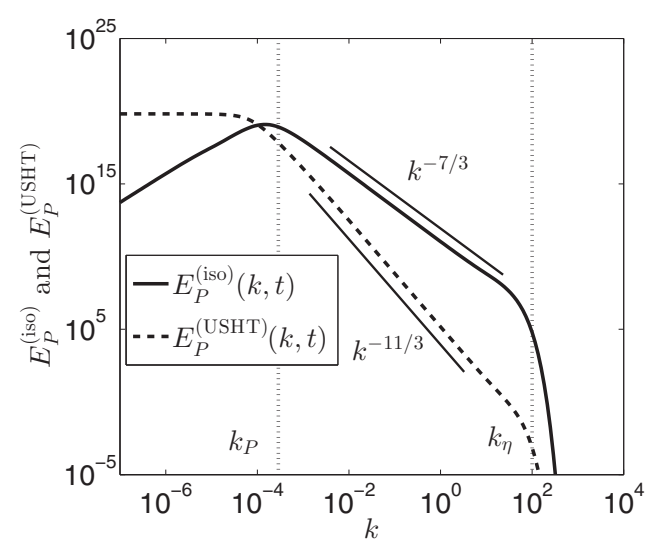

(a)

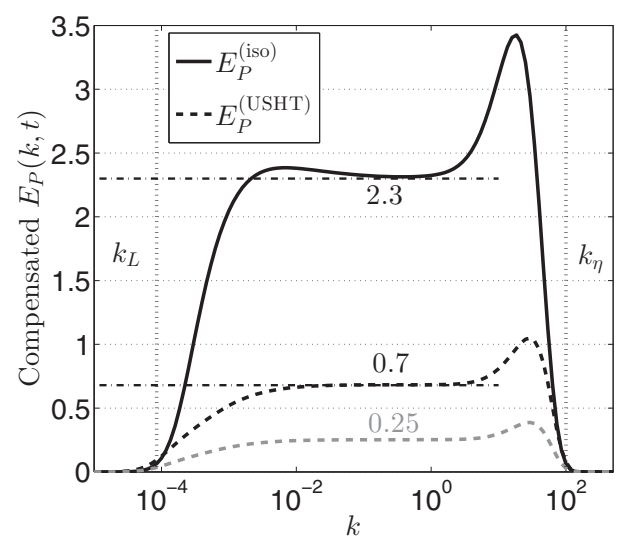

(b)

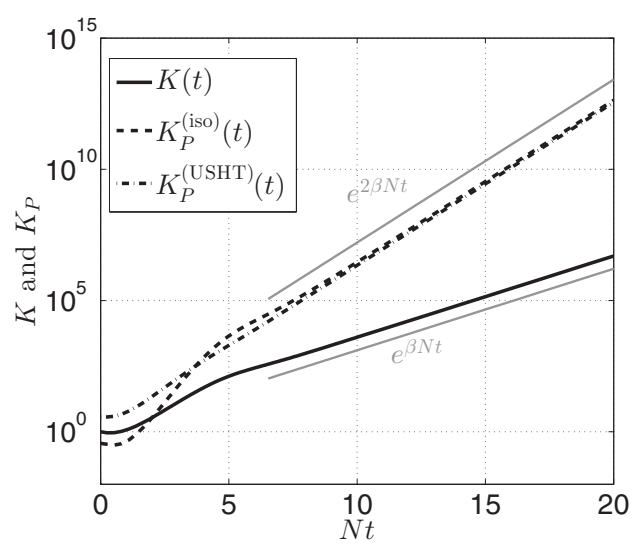

(c)

FIG. 14. Pressure spectra in USHT for $\sigma=2$ at $\operatorname{Re}_{\lambda}=2 \times 10^{4}$ : (a) isotropic and anisotropic pressure spectra $E_{P}^{(\text {iso) }}$ and $E_{P}^{(\text {USHT) }}$, along with the pressure integral wave number $k_{P}$ and the Kolmogorov wave number $k_{\eta}$, at $\operatorname{Re}_{\lambda} \simeq 3 \times 10^{4}$; (b) compensated pressure spectra $E_{P}^{\text {(iso) }} k^{7 / 3} \epsilon^{-4 / 3}$ and $E_{P}^{(\text {USHT) }} k^{11 / 3} \epsilon^{-2 / 3} / N^{2}$ in black and $E_{P}^{\text {(USHT) }} k^{11 / 3} \epsilon^{1 / 3} / \epsilon_{T} N^{2}$ in gray; and (c) isotropic and anisotropic parts $K_{P}^{\text {(iso) }}$ and $K_{P}^{\text {(USHT) }}$ of the pressure variance, along with the kinetic energy $K$. The gray lines indicate the curves $\exp (\beta N t)$ and $\exp (2 \beta N t)$.

is investigated as well. Both are found to grow exponentially at a rate $2 \gamma$ in Fig. 13(c), where $\gamma$ is the exponential growth rate of the kinetic energy $K(t)$ in shear flows, in agreement with theoretical predictions [36].

Now the framework of USHT is addressed. In Fig. 14(a) the scaling of the isotropic and anisotropic parts of $E_{P}$ are presented. The turbulence-turbulence interaction spectrum scales in $E_{P}^{(\text {iso) }} \sim k^{-7 / 3}$ : The constant $C_{P}=2.3$ in Fig. 14(b) is close to the value obtained in shear flows, which indicates some universality of the isotropic pressure spectrum. Then the anisotropic part resulting from stratification is presented in Fig. 14(a) and scales in $E_{P}^{(\mathrm{USHT})} \sim k^{-11 / 3}$. The $k^{-11 / 3}$, analogous to the anisotropic part in shear flow, is expected from the expression (63), because $E_{T} \sim k^{-5 / 3}$ in the inertial range. Similarly to shear flows, the anisotropic part has a quadratic dependence on the mean-field gradient, given its expression (63). Thus, only the dependence on the dissipation rates remains to be determined. Since the scalar field is rescaled as a buoyant velocity, there are infinite possibilities of the form $\epsilon^{a} \epsilon_{T}^{b}$, with $a+b=2 / 3$, by dimensional analysis. One could choose the 
inertial scaling of $E_{T}$, i.e., $a=-1 / 3$ and $b=1$, but this yields in Fig. 14(b) (in gray) a constant quite small of order 0.25 ; this is not satisfactory, since for shear flows the constant is very close to Kolmogorov. Given the similarities pointed out so far, we choose $a=2 / 3$ and $b=0$, as for $E_{P}^{(\mathrm{S})}$, which provides in Fig. 14(b) (in black) a constant $C_{P}^{(\text {USHT) }} \simeq 0.7$. This seems more relevant because it is close to the Corrsin-Obukhov constant $\left(K_{\mathrm{CO}}=0.76\right)$, consistently with $E_{P}^{(\mathrm{USHT})}$ depending on $E_{T}$. In the end, the scaling of the anisotropic part of the pressure spectrum in USHT reads

$$
E_{P}^{(\mathrm{USHT})}(k, t)=C_{P}^{(\mathrm{USHT})} N^{2} \epsilon^{2 / 3} k^{-11 / 3}, \quad C_{P}^{(\mathrm{USHT})} \simeq 0.7 .
$$

This scaling is a fundamental result, interesting for two reasons: First, it is strongly analogous to the scaling of the anisotropic part of the pressure spectrum in shear flows $\left(\epsilon^{2 / 3} k^{-11 / 3}\right)$; second, $C_{P}^{\text {(USHT) }}$ is close to the Corrsin-Obukhov constant, similarly to $C_{P}^{(\mathrm{S})}$ being close to the Kolmogorov one.

Finally, the time evolution of the isotropic and anisotropic parts $K_{P}^{(\text {iso) }}$ and $K_{P}^{(\mathrm{USHT})}$ of the pressure variance are displayed in Fig. 14(c). It is found, similarly to shear flows, that the pressure variances grow exponentially at a rate $2 \beta$, where $\beta$ is the exponential growth rate of the kinetic energy. Interestingly, the exponential growth rate of the pressure variance still depends on the infrared slope $\sigma$ of the kinetic energy spectrum, even though the infrared slope of the isotropic pressure spectrum is $E_{P}^{(\text {iso) }} \sim k^{2}$.

In conclusion, there is a strong analogy between pressure spectra in shear flows and unstably stratified turbulence.

\section{B. Cospectrum at high Schmidt numbers}

In this section the case of a weakly diffusive active scalar with Sc $\gg 1$ is addressed. This is representative of the example described in the Introduction, i.e., unstably stratified water columns in the ocean generated by double diffusion mechanisms. For HIT, it has been known since the pioneering work of Batchelor [39] that beyond the Kolmogorov wave number $k_{\eta}$, scalar fluctuations are convected by the velocity field of the Kolmogorov scale up to the Batchelor wave number $k_{B}=\sqrt{S c} k_{\eta}$, with a characteristic time scale $\sqrt{v / \epsilon}$, thus forming the so-called viscous-convective range where the scalar variance spectrum scales as

$$
E_{T}(k, t)=K_{B} \epsilon_{T} \sqrt{\frac{\nu}{\epsilon}} k^{-1},
$$

where $K_{B}$ is the Batchelor constant, found to be $\simeq 2.5$ in the present simulations. Despite the difficulty, especially in DNS, to solve the very small scales, the $k^{-1}$ range was recovered several times in HITSG where the passive scalar fluctuations are sustained by a mean scalar gradient $[40,41]$. In purely isotropic turbulence, the $k^{-1}$ range was also recovered recently by the EDQNM model [34,42] at very large Reynolds numbers. It is worth noting that because of the logarithmic discretization, nonlocal scalar transfers from large to very small scales, corresponding to elongated triads such that $q \ll k \sim p$, have to be added to the isotropic nonlinear transfer $S^{\mathrm{T}, \mathrm{NL}(\mathrm{iso})}$. Theoretical details and validation of these nonlocal transfers in HIT can be found in Refs. [10,34,42]. Numerically, it is found that one term of the expansion dominates the others, so the direct scalar nonlocal transfer reads

$$
T_{T}^{+}(k, t)=-\frac{\partial}{\partial k}\left[\frac{2}{15} k\left(2 E_{T}(k)-k \frac{\partial E_{T}}{\partial k}\right) \int_{0}^{\tilde{a} k} \theta_{k k q}^{T} q^{2} E(q) d q\right],
$$

where the scalar eddy-damping term is recalled in the Appendix and $\tilde{a}$ is the nonlocal parameter, which is here $\tilde{a}=10^{1 / 15}-1=0.1659$. The impact of this nonlocal transfer is illustrated in Fig. 15(a), where the fluxes of $S^{\mathrm{T}, \mathrm{NL}(\mathrm{iso})}$ and $T_{T}^{+}$are respectively denoted by $\Pi_{T}^{(\text {iso) }}$ and $\Pi_{T}^{+}$. It is clear that the nonlocal transfer, which is conservative since $\Pi_{T}^{+}(k=0)=\Pi_{T}^{+}\left(k>k_{B}\right)=0$, brings energy beyond the Kolmogorov wave number in the viscous-convective range. Consequently, in this 


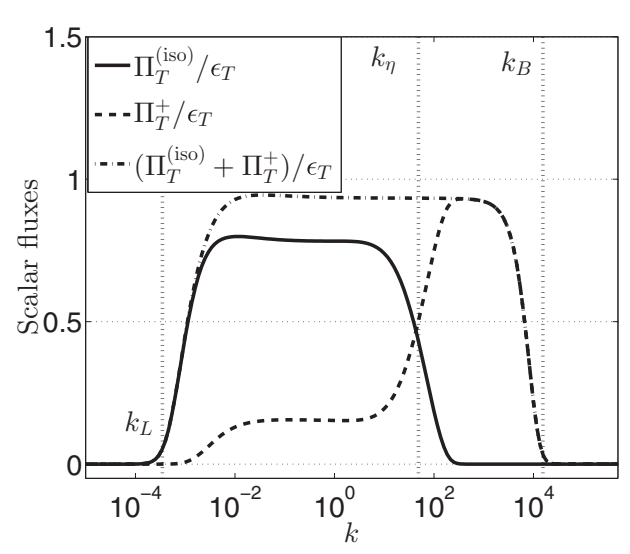

(a)

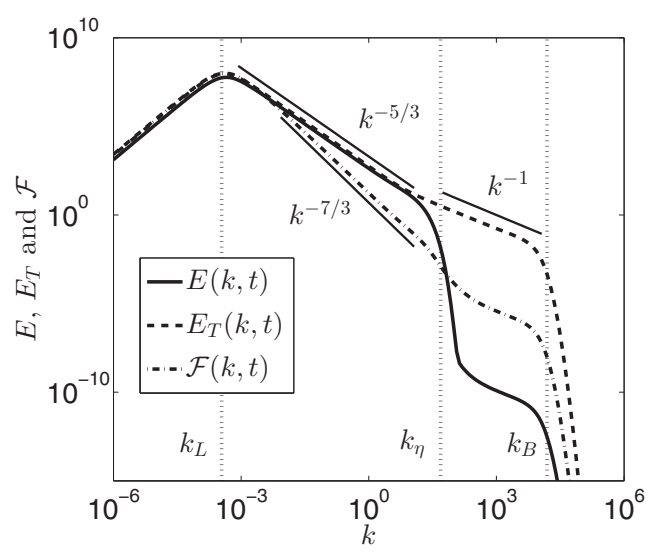

(b)

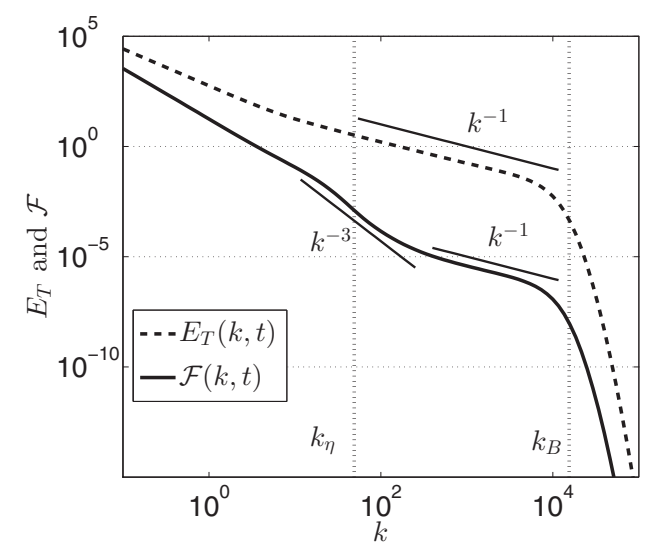

(c)

FIG. 15. Saffman USHT for $\mathrm{Sc}=10^{5}$ at $\operatorname{Re}_{\lambda}=10^{4}$, along with the integral, Kolmogorov, and Batchelor wave numbers $k_{L}, k_{\eta}$, and $k_{B}$, respectively: (a) normalized fluxes $\Pi_{T}^{\text {(iso) }}$ and $\Pi_{T}^{+}$of $S^{\mathrm{T}, \mathrm{NL}(\text { iso) }}$ and $T_{T}^{+}$; (b) $E$, $E_{T}$, and $\mathcal{F}$, along with the $k^{-5 / 3}$ inertial scaling for $E$ and $E_{T}$, the $k^{-7 / 3}$ inertial scaling for $\mathcal{F}$, and the $k^{-1}$ viscous-convective scaling for $E_{T}$; and (c) zoom in of the viscous-convective range for $E_{T}$ and $\mathcal{F}$, with different scalings explained in the text.

section, the nonlocal transfer $T_{T}^{+}$is added to the nonlinear transfer $S^{\mathrm{T}, \mathrm{NL}(\mathrm{iso})}$ in the evolution equation of $E_{T}$.

The scalar flux $\mathcal{F}$ for Sc $\gg 1$ was investigated in HITSG in Ref. [19] and found to decrease sharply after $k_{\eta}$, similarly to the kinetic energy spectrum. This feature was recovered with the present anisotropic EDQNM modeling in Ref. [43]. It appears that in USHT, the behavior of the cospectrum beyond $k_{\eta}$ is completely different, as revealed in Fig. 15(b): Indeed, unlike passive scalar dynamics, the scalar flux survives in the viscous-convective range. The buoyant spectrum $E_{T}$ still scales in $k^{-1}$ and there are also small-scale fluctuations for $E$ beyond $k_{\eta}$, but they are much less intense than for $E_{T}$ and $\mathcal{F}$, so they are neglected.

The scaling of $\mathcal{F}$ in the viscous-convective range is not clear, but it is close to $k^{-1}$, slightly steeper, as revealed in Fig. 15(c). Around $k_{\eta}$, the cospectrum seems to scale in $k^{-3}$ on a decade, but this is very likely just a transition toward the viscous-convective scaling. Nevertheless, both the $k^{-1}$ and $k^{-3}$ scalings can be briefly justified using classical arguments. The $k^{-3}$ scaling can be obtained by assuming that at small scales where $E_{T} \sim k^{-1}$ there is a balance between viscous dissipation and 


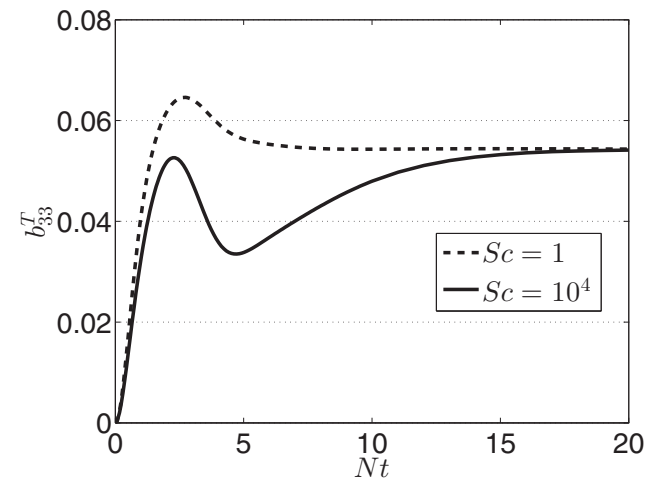

(a)

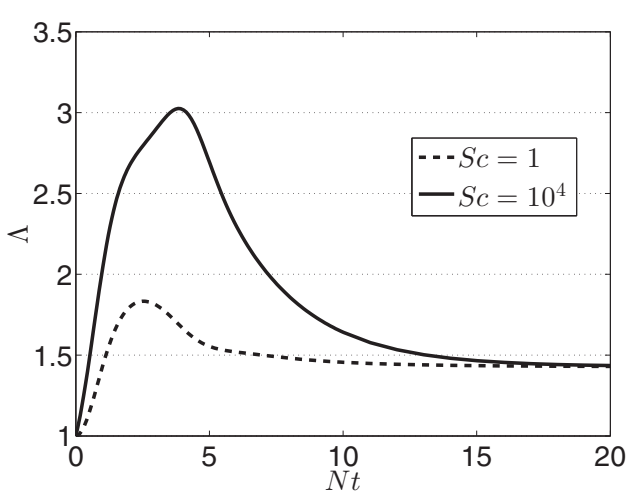

(b)

FIG. 16. Saffman USHT for $\mathrm{Sc}=1$ and $\mathrm{Sc}=10^{4}$ : (a) scalar anisotropy indicator $b_{33}^{T}$ and (b) mixing intensity $\Lambda$.

production of buoyant fluctuations in Eq. (33), so

$$
(v+a) k^{2} \mathcal{F} \sim \frac{2}{3} N E_{T} \Leftrightarrow \mathcal{F}(k, t) \sim N \frac{\epsilon_{T}}{\sqrt{v \epsilon}} k^{-3} .
$$

In this expression, $E_{T} H_{33}^{(T)}$ was neglected compared to $E_{T}$. For reasons that are explained hereafter, it is preferred to express this scaling as

$$
\mathcal{F}(k, t) \sim N \sqrt{\frac{\epsilon}{v}} \frac{\epsilon_{T}}{\epsilon} k^{-3},
$$

where the inverse of the Kolmogorov time scale $\sqrt{v / \epsilon}$ appears, consistently with the dynamics of the viscous-convective range. Then, after $k_{\eta}$, the scaling is slightly steeper than $k^{-1}$, but nevertheless the Batchelor scaling seems relevant if one assumes, as for $E_{T}$, that the characteristic time scale of $\mathcal{F}$ in the viscous-convective range is also independent of $k$. Since this range exists only due to the small-scale coupling through $N$, it makes sense to assume that it depends linearly on $N$ and also on $\epsilon$ and $v$; this provides $N v / \epsilon$ as the characteristic time scale of the cospectrum in the viscous-convective range. Further assuming that $\mathcal{F}$ depends only on this time scale, $k$, and $\epsilon_{T}$ yields

$$
\mathcal{F}(k, t) \sim \frac{N v}{\epsilon} \epsilon_{T} k^{-1} .
$$

It is worth noting that, unlike $E_{T}$ for which nonlocal transfers are at the origin of the viscous convective range, the range for $\mathcal{F}$ beyond $k_{\eta}$ is created by local production of buoyant fluctuations through the term $N E_{T}$. These scalings are original results for high-Schmidt-number USHT, which could be of interest for turbulent models in oceanography, for instance, but would still deserve some confirmation by DNS.

The change from the scaling in $k^{-3}$, around $k_{\eta}$, to $k^{-1}$, just after $k_{\eta}$, can be understood in terms of characteristic time scales. For the scaling (74), the characteristic time is $\sqrt{\nu / \epsilon}$, which is the classical characteristic time of the Kolmogorov scale. For smaller scales, viscous dissipation becomes more and more important, so the characteristic time evolves from $\sqrt{v / \epsilon}$ toward $\left(\nu k^{2}\right)^{-1}$, which directly yields (75). Then the characteristic time scale saturates to $N v / \epsilon$. These two scalings and their characteristic times are consistent with the Kolmogorov scale being the wave number around which the cospectrum changes from $k^{-3}$ to $k^{-1}$. Indeed, equating (74) and (75) yields $k=k_{\eta}$.

Regarding the one-point statistics, obviously, even with high Schmidt numbers, simulations show that the asymptotic anisotropic state still depends on $\sigma$ and not on $N$ and the exponential growth rate $\beta$ is not modified with respect to the case $\mathrm{Sc}=1$. Nevertheless, illustrated in Fig. 16 is the impact 
of a large Sc on the early dynamics of the scalar anisotropy indicator $b_{33}^{T}$ and the mixing parameter $\Lambda$ (the effects are negligible for Fr and $b_{33}$ ). The main result is that a large Sc does not change the asymptotic values with respect to the case $\mathrm{Sc}=1$. However, specifically for $b_{33}^{T}$ and $\Lambda$, which depend explicitly on the scalar field unlike $b_{33}$ and Fr, a large Schmidt number strongly impacts the transient regime as well: $\Lambda$ initially increases much more with $\mathrm{Sc}=10^{4}$ than with $\mathrm{Sc}=1$, whereas $b_{33}^{T}$ is always smaller at $\mathrm{Sc}=10^{4}$.

At $\mathrm{Sc}=10^{4}$, even if there is no inertial range initially for $E_{T}$, the viscous convective range spans almost two decades. This is completely different from the case $\mathrm{Sc}=1$. This initial viscous-convective range thus contributes greatly to $\Lambda$ because it gives large initial values of $K_{T}$, which explains the strong increase at small $N t$. Then, when the Reynolds number increases, the inertial ranges of $E$ and $E_{T}$ become dominant in the integrals for $K$ and $K_{T}$, so eventually the same asymptotic value as for $\mathrm{Sc}=1$ is recovered. In contrast, for $b_{33}^{T}$, the viscous-convective range adds isotropic small scales, thus reducing initially the global anisotropy over the whole wave-number space.

In conclusion, unlike passive scalar dynamics, the cospectrum survives in the viscous-convective range for USHT for large Schmidt numbers and scales in $k^{-1}$, similarly to the scalar variance spectrum, after a transient $k^{-3}$ subrange around the Kolmogorov wave number. Finally, large Schmidt numbers strongly affect the early dynamics of $b_{33}^{T}$ and $\Lambda$, nevertheless without changing the asymptotic state.

\section{CONCLUSION}

Unstably stratified homogeneous turbulence was investigated numerically at large Reynolds numbers with an anisotropic EDQNM modeling, originally derived for passive scalar dynamics [14] and consistently extended here to the framework of active scalar dynamics, which is the first theoretical contribution of this work. In the part consisting of numerical results at large Reynolds numbers, comparisons were made with the axisymmetric EDQNM model developed by Burlot and co-workers [4-6], where the production terms were treated exactly with no modeling of anisotropy and the nonlinear transfers were closed with a classical EDQNM procedure. Moreover, since our modeling was applied in previous work for different configurations (notably, transport of passive scalar dynamics in isotropic turbulence with a mean scalar gradient and shear flows [12-14]) qualitative comparisons were also made with these cases and some interesting differences and similarities were found between shear-driven flows and unstably stratified turbulence.

The time evolution of the kinetic energy, scalar variance (or buoyancy), and scalar flux spectra $E(k, t), E_{T}(k, t)$, and $\mathcal{F}(k, t)$ were addressed: The $k^{-5 / 3}$ inertial scaling of $E$ and $E_{T}$ was recovered, along with the $k^{-7 / 3}$ inertial scaling of the cospectrum $\mathcal{F}$. For the latter compensated spectrum, a plateau starts appearing at the highest Reynolds numbers reached here $\left(\operatorname{Re}_{\lambda} \sim 10^{6}\right)$. The $k^{-3}$ time evolution of the peak of the three previous spectra was also recovered and justified. For the infrared dynamics, it was found that because of the strong coupling between $E, E_{T}$, and $\mathcal{F}$ due to stratification, the spectrum with initially the smallest infrared slope $\sigma$ [where $E_{(T)}\left(k<k_{L}, t\right) \sim k^{\sigma_{(T)}}$ ] imposes the minimum energy on the others, which significantly differs from the passive scalar dynamics.

Then the effects of varying the stratification frequency $N$ and the infrared slope $\sigma$ on the asymptotic anisotropic states of one-point statistics in USHT were studied, specifically the Froude number Fr, the mixing intensity $\Lambda$, global anisotropy indicators for the velocity and scalar fields $b_{33}$ and $b_{33}^{T}$, and the exponential growth rate $\beta$ of the kinetic energy, scalar variance, and mixed correlation. The conclusion is, in agreement with the axisymmetric EDQNM results, that the asymptotic states of these quantities strongly depend on $\sigma$. This feature is completely different from shear flows where one-point statistics no longer depend on $\sigma$ asymptotically, at least within the same modeling. However, for both shear flows and USHT, varying the mean gradient intensity impacts only short times. In particular, it was found that the more energy there is initially in large scales, i.e., the smaller $\sigma$ is, the more anisotropic the flow is. Furthermore, at large Reynolds numbers, anisotropy is gathered at large scales, whereas small scales return to isotropy for both the velocity and buoyancy fields (at least at the level of second-order moments). Satisfactory agreement is found 
in the quantitative comparison with the axisymmetric EDQNM model; nevertheless, one can point out some differences between the two approaches. (i) With our anisotropic EDQNM modeling, the flow is less anisotropic than in Refs. [4-6], meaning, notably, that our anisotropy indicator $b_{33}$ is slightly smaller. (ii) A theoretical prediction for the exponential growth rate $\beta$ of kinetic energy was proposed, based on the linear operator of our evolutions equations, and assessed numerically. Whatever the large-scale initial conditions $\sigma$ are, our growth rate is $10 \%$ smaller than the one of [4,5], consistently with our flow being less anisotropic. (iii) The Froude number is higher with the anisotropic EDQNM modeling, but can be decreased to values very close to those of [4-6] by changing the eddy-damping constants, as shown in the Appendix.

These discrepancies are probably due to the modeling of anisotropy in our approach. Indeed, we use an expansion into spherical harmonics truncated at the second order of the spectral correlations. This truncation and its possible consequences were discussed in detail in Refs. [12-14]. Even though it seems that the truncation may be the reason for the flow to be less anisotropic here, it does not prevent the present modeling from recovering all the important features of USHT recalled just before: notably, the dependence of asymptotic states on large-scale initial conditions $\sigma$.

Finally, two applications of our anisotropic EDQNM modeling were proposed, which constitute fundamental results. First, pressure spectra in USHT were investigated and it was found that the anisotropic part, resulting from stratification, scales in $k^{-11 / 3}$ in the inertial range, whereas the isotropic part scales in $k^{-7 / 3}$; these scalings are completely similar to pressure spectra in shear flows. It was also shown that the pressure variance grows exponentially at a rate $2 \beta$, where $\beta$ is the growth rate of the kinetic energy. Then high Schmidt numbers were considered. The scalar variance spectrum still scales in $k^{-1}$ in the viscous-convective range beyond the Kolmogorov wave number, as in HIT. The main result here is that the cospectrum, which was strongly decreasing in this region for passive scalar dynamics, now also displays a viscous-convective range with a scaling close to $k^{-1}$, after a transient $k^{-3}$ subrange around $k_{\eta}$. It is worth noting that large Schmidt numbers do not affect the asymptotic values of one-point statistics or the exponential growth rate $\beta$, but only the transient regime of $\Lambda$ and $b_{33}^{T}$.

In conclusion, the present anisotropic EDQNM modeling permits one to investigate largeReynolds-number asymptotic anisotropic states of USHT, with small computational resources. The main features of the dynamics studied by Burlot and co-workers were recovered and fundamental results were derived for the case of large Schmidt numbers. Since the present modeling is quite versatile, e.g., it can handle both passive and active scalar dynamics, shear flows, axisymmetric contractions, distortion [12-14], it could be applied in future works, for instance, to unstably stratified shear flows. Early simulations seem to indicate that the exponential growth $\beta$, with $K \sim e^{\beta N t}$, still depends on $\sigma$ with the presence of shear, but also on $N$ and $S$, and more precisely that $\beta$ decreases toward the USHT predictions with higher $N$ at fixed $\sigma$ and $S$.

\section{ACKNOWLEDGMENT}

The authors acknowledge fruitful discussions with B.-J. Gréa and O. Soulard, which greatly helped improve this work.

\section{APPENDIX: EFFECTS OF A DIFFERENT CHOICE OF EDDY-DAMPING CONSTANTS}

In this Appendix we briefly discuss the impact of changing the eddy-damping constants on the dynamics of USHT. First, we recall that for consistency with the development of the present anisotropic EDQNM modeling in Ref. [14], the same eddy-damping constants are kept here for the extension to the case of active scalar dynamics, i.e., $A_{1}=0.355, A_{2}=0$, and $A_{3}=1.3$ (EDC1), where $A_{1}$ is for the velocity field and $A_{2}$ and $A_{3}$ are for the scalar field. The EDC1 setting was consequently kept throughout the paper, except in Sec. III B 5, where a quantitative comparison was performed against the axisymmetric EDQNM model of Burlot and co-workers [5]. Indeed, in the latter work, a different choice of eddy-damping constants was made, i.e., $A_{1}=A_{2}=A_{3}=0.355$ 


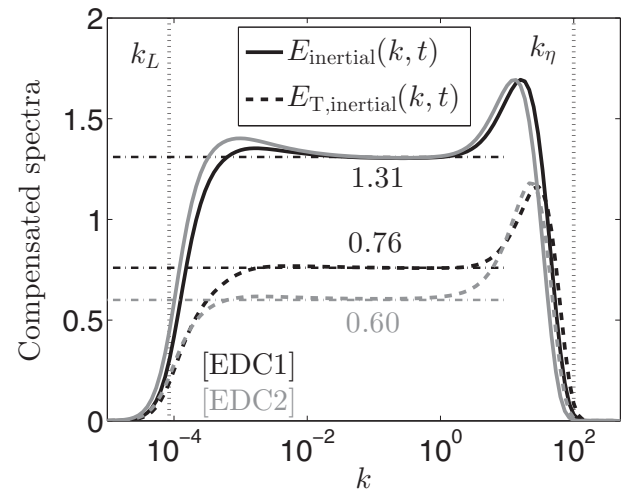

(a)

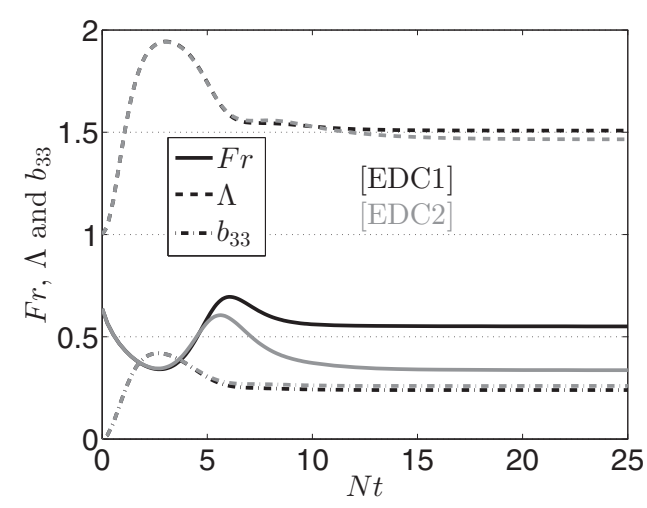

(b)

FIG. 17. Comparisons of the eddy-damping constant settings in Saffman turbulence with $A_{1}=0.355$, $A_{2}=0$, and $A_{3}=1.3$ (EDC1) (black lines) and $A_{1}=A_{2}=A_{3}=0.355$ (EDC2) (gray lines). (a) Kinetic energy and scalar variance compensated spectra with $\operatorname{Re}_{\lambda}(N t=20)=3 \times 10^{4}$. The solid line is for $E k^{5 / 3} \epsilon^{-2 / 3}$ and the dashed line is for $E_{T} k^{5 / 3} \epsilon_{T}^{-1} \epsilon^{1 / 3}$. (b) Plot of Fr, $\Lambda$, and $b_{33}$.

(EDC2). Furthermore, in Ref. [4], a correction to the eddy-damping term is added to match with DNS; this is not considered here. Indeed, simulations not presented here have revealed that such an isotropized correction has only a slight impact on the early dynamics, which is already satisfactorily captured as shown in Fig. 10, and does not modify the exponential growth rate $\beta$.

For clarity, the expressions of the different eddy-damping terms $\theta_{k p q}, \theta_{k p q}^{T}$, and $\theta_{k p q}^{F}$ for the velocity, scalar, and scalar flux are given by

$$
\begin{aligned}
\theta_{k p q} & =\frac{1-\exp \left\{-\left[v\left(k^{2}+p^{2}+q^{2}\right)+\mu_{1}(k, t)+\mu_{1}(p, t)+\mu_{1}(q, t)\right] t\right\}}{v\left(k^{2}+p^{2}+q^{2}\right)+\mu_{1}(k, t)+\mu_{1}(p, t)+\mu_{1}(q, t)}, \\
\theta_{k p q}^{T} & =\frac{1-\exp \left\{-\left[a\left(k^{2}+p^{2}\right)+v q^{2}+\mu_{2}(k, t)+\mu_{2}(p, t)+\mu_{3}(q)\right] t\right\}}{a\left(k^{2}+p^{2}\right)+v q^{2}+\mu_{2}(k, t)+\mu_{2}(p, t)+\mu_{3}(q, t)}, \\
\theta_{k p q}^{F} & =\frac{1-\exp \left\{-\left[a k^{2}+v\left(p^{2}+q^{2}\right)+\mu_{2}(k, t)+\mu_{3}(p, t)+\mu_{3}(q, t)\right] t\right\}}{a k^{2}+v\left(p^{2}+q^{2}\right)+\mu_{2}(k, t)+\mu_{3}(p, t)+\mu_{3}(q, t)},
\end{aligned}
$$

where

$$
\mu_{i}(k, t)=A_{i} \sqrt{\int_{0}^{k} k^{\prime 2} E\left(k^{\prime}, t\right) d k^{\prime}}, \quad i=1,2,3 .
$$

It is recalled that these eddy-damping terms can be seen as the characteristic time of their corresponding triple correlations.

With the present setting EDC1, the Kolmogorov and Corrsin-Obukhov constants are consistent with those obtained for passive scalar dynamics, $K_{0}=1.31$ and $K_{\mathrm{CO}}=0.76$, as presented in Sec. III A. Choosing EDC2 as in Refs. [4,5] tends first to decrease $K_{\mathrm{CO}}$ to values smaller than usual ones $\left(K_{\mathrm{CO}}=0.6\right)$, as revealed in Fig. 17(a), whereas $K_{0}$ remains unchanged. Furthermore, with EDC2, the flow is slightly more anisotropic in Fig. 17(b). Indeed, $b_{33}$ increases a bit from EDC1 to EDC2. The main difference is observed in Fr, which is reduced with EDC2. This means that the latter choice of eddy-damping constants enhances the importance of stratification in the dynamics, without increasing significantly the global anisotropy.

Hence, changing the eddy-damping constants from EDC1 to EDC2 slightly increases the global anisotropy of the flow and reduces Fr, which makes our results closer to [4,5]. However, the counterpart is a decrease of the Corrsin-Obukhov constant, which is another reason why EDC1 is 
preferred in this paper. Moreover, whether EDC1 or EDC2 is chosen, it does not improve the plateau for the cospectrum $\mathcal{F}$ or change the exponential growth rate $\beta$ of the kinetic energy.

[1] D. H. Sharp, An overview of Rayleigh-Taylor instability, Physica D 3, 3 (1984).

[2] O. Soulard and J. Griffond, Inertial-range anisotropy in Rayleigh-Taylor turbulence, Phys. Fluids 24, 025101 (2012).

[3] O. Soulard, J. Griffond, and B.-J. Gréa, Large-scale analysis of self-similar unstably stratified homogeneous turbulence, Phys. Fluids 26, 015110 (2014).

[4] A. Burlot, B.-J. Gréa, F. S. Godeferd, C. Cambon, and J. Griffond, Spectral modeling of high Reynolds number unstably stratified homogeneous turbulence, J. Fluid Mech. 765, 17 (2015).

[5] A. Burlot, B.-J. Gréa, F. S. Godeferd, C. Cambon, and O. Soulard, Large Reynolds number self-similar states of unstably stratified homogeneous turbulence, Phys. Fluids 27, 065114 (2015).

[6] B.-J. Gréa, A. Burlot, F. Godeferd, J. Griffond, O. Soulard, and C. Cambon, Dynamics and structure of unstably stratified homogeneous turbulence, J. Turbul. 17, 651 (2016).

[7] D. Livescu and J. R. Ristorcelli, Buoyancy-driven variable-density turbulence, J. Fluid Mech. 591, 43 (2007).

[8] D. Chung and D. I. Pullin, Direct numerical simulation and large-eddy simulation of stationary buoyancydriven turbulence, J. Fluid Mech. 643, 279 (2010).

[9] S. A. Orszag, Analytical theories of turbulence, J. Fluid Mech. 41, 363 (1970).

[10] M. Lesieur, Turbulence in Fluids, 4th ed., Fluid Mechanics and its applications Vol. 84 (Springer Netherlands, Dordrecht, 2008).

[11] C. Cambon and R. Rubinstein, Anisotropic developments for homogeneous shear flows, Phys. Fluids 18, 085106 (2006).

[12] V. Mons, C. Cambon, and P. Sagaut, A spectral model for homogeneous shear-driven anisotropic turbulence in terms of spherically-averaged descriptors, J. Fluid Mech. 788, 147 (2016).

[13] A. Briard, T. Gomez, V. Mons, and P. Sagaut, Decay and growth laws in homogeneous shear turbulence, J. Turbul. 17, 699 (2016).

[14] A. Briard, T. Gomez, and C. Cambon, Spectral modeling for passive scalar dynamics in homogeneous anisotropic turbulence, J. Fluid Mech. 799, 159 (2016).

[15] A. Briard and T. Gomez, Dynamics of helicity in homogeneous skew-isotropic turbulence, J. Fluid Mech. (to be published).

[16] C. Cambon and L. Jacquin, Spectral approach to non-isotropic turbulence subjected to rotation, J. Fluid Mech. 202, 295 (1989).

[17] C. Cambon, N. M. Mansour, and F. Godeferd, Energy transfer in rotating turbulence, J. Fluid Mech. 337, 303 (1997).

[18] C. Cambon, L. Danaila, F. S. Godeferd, and J. F. Scott, Third-order statistics and the dynamics of strongly anisotropic turbulent flows, J. Turbul. 14, 121 (2013).

[19] P. A. O'Gorman and D. I. Pullin, Effect of Schmidt number on the velocity-scalar cospectrum in isotropic turbulence with a mean scalar gradient, J. Fluid Mech. 532, 111140 (2005).

[20] D. K. Maiti, A. S. Gupta, and S. Bhattacharyya, Stable/unstable stratification in thermosolutal convection in a square cavity, J. Heat Transfer 130, 122001 (2008).

[21] D. M. Sigman, S. L. Jaccard, and G. H. Haug, Polar ocean stratification in a cold climate, Nature (London) 428, 59 (2004).

[22] P. Sagaut and C. Cambon, Homogeneous Turbulence Dynamics (Cambridge University Press, New York, 2008).

[23] S. Herr, L.-P. Wang, and L. R. Collins, EDQNM model of a passive scalar with a uniform mean gradient, Phys. Fluids 8, 1588 (1996).

[24] W. J. T. Bos, H. Touil, and J.-P. Bertoglio, Reynolds number dependency of the scalar flux spectrum in isotropic turbulence with a uniform scalar gradient, Phys. Fluids 17, 125108 (2005). 
[25] J. R. Herring, Approach of axisymmetric turbulence to isotropy, Phys. Fluids 17, 859 (1974).

[26] C. Cambon, V. Mons, B.-J. Gréa, and R. Rubinstein, Anisotropic triadic closures for shear-driven and buoyancy-driven turbulent flows, Comput. Fluids, doi:10.1016/j.compfluid.2016.12.006.

[27] S. B. Pope, Turbulent Flows (Cambridge University Press, Cambridge, 2000).

[28] J. R. Herring, D. Schertzer, M. Lesieur, G. R. Newman, J. P. Chollet, and M. Larcheveque, A comparative assessment of spectral closures as applied to passive scalar diffusion, J. Fluid Mech. 124, 411 (1982).

[29] O. Poujade, Rayleigh-Taylor Turbulence is Nothing Like Kolmogorov Turbulence in the Self-Similar Regime, Phys. Rev. Lett. 97, 185002 (2006).

[30] J. L. Lumley, Similarity and the turbulent energy spectrum, Phys. Fluids 10, 855 (1967).

[31] V. M. Canuto, M. S. Dubovikov, and A. Dienstfrey, A dynamical model for turbulence. IV. Buoyancydriven flows, Phys. Fluids 9, 2118 (1997).

[32] M. Lesieur and S. Ossia, 3D isotropic turbulence at very high Reynolds numbers: EDQNM study, J. Turbul. 1, 1 (2000).

[33] M. Meldi and P. Sagaut, Further insights into self-similarity and self-preservation in freely decaying isotropic turbulence, J. Turbul. 14, 24 (2013).

[34] A. Briard, T. Gomez, P. Sagaut, and S. Memari, Passive scalar decay laws in isotropic turbulence: Prandtl number effects, J. Fluid Mech. 784, 274 (2015).

[35] A. J. Simonsen and P.-A. Krogstad, Turbulent stress invariant analysis: Clarification of existing terminology, Phys. Fluids 17, 088103 (2005).

[36] W. K. George, P. D. Beuther, and R. E. A. Arndt, Pressure spectra in turbulent free shear flows, J. Fluid Mech. 148, 155 (1984).

[37] M. Meldi and P. Sagaut, Pressure statistics in self-similar freely decaying isotropic turbulence, J. Fluid Mech. 717, R2-1 (2013).

[38] M. Lesieur, S. Ossia, and O. Métais, Infrared pressure spectra in two- and three-dimensional isotropic incompressible turbulence, Phys. Fluids 11, 1535 (1999).

[39] G. K. Batchelor, Small-scale variation of convected quantities like temperature in turbulent fluid Part 1. General discussion and the case of small conductivity, J. Fluid Mech. 5, 113 (1959).

[40] P. K. Yeung, S. Xu, and K. R. Sreenivasan, Schmidt number effects on turbulent transport with uniform mean scalar gradient, Phys. Fluids 14, 4178 (2002).

[41] P. K. Yeung, S. Xu, D. A. Donzis, and K. R. Sreenivasan, Simulations of three-dimensional turbulent mixing for schmidt numbers of the order 1000, Flow Turbul. Combust. 72, 333347 (2004).

[42] A. Briard and T. Gomez, Mixed-derivative skewness for high Prandtl and Reynolds numbers in homogeneous isotropic turbulence, Phys. Fluids 28, 081703 (2016).

[43] A. Briard and T. Gomez, Prandtl number effects in decaying homogeneous isotropic turbulence with a mean scalar gradient, J. Turbul. 18, 418 (2017). 